Moroccan J. of Pure and Appl. Anal. (MJPAA)

Volume 7(2), 2021, Pages 182-213

ISSN: Online 2351-8227 - Print 2605-6364

DOI: $10.2478 / \mathrm{mjpaa}-2021-0014$

\title{
Optimized Schwarz methods with general Ventcell transmission conditions for fully anisotropic diffusion with discrete duality finite volume discretizations
}

\author{
Martin J. Gander ${ }^{1}$, Laurence Halpern ${ }^{2}$, Florence Hubert ${ }^{3}$, and Stella Krell ${ }^{4}$
}

\begin{abstract}
AвstRACT. We introduce a new non-overlapping optimized Schwarz method for fully anisotropic diffusion problems. Optimized Schwarz methods take into account the underlying physical properties of the problem at hand in the transmission conditions, and are thus ideally suited for solving anisotropic diffusion problems. We first study the new method at the continuous level for two subdomains, prove its convergence for general transmission conditions of Ventcell type using energy estimates, and also derive convergence factors to determine the optimal choice of parameters in the transmission conditions. We then derive optimized Robin and Ventcell parameters at the continuous level for fully anisotropic diffusion, both for the case of unbounded and bounded domains. We next present a discretization of the algorithm using discrete duality finite volumes, which are ideally suited for fully anisotropic diffusion on very general meshes. We prove a new convergence result for the discretized optimized Schwarz method with two subdomains using energy estimates for general Ventcell transmission conditions. We finally study the convergence of the new optimized Schwarz method numerically using parameters obtained from the continuous analysis. We find that the predicted optimized parameters work very well in practice, and that for certain anisotropies which we characterize, our new bounded domain analysis is important.
\end{abstract}

Mathematics Subject Classification (2020). 65N55, 65N08, 35A35, 65F10.

Key words and phrases. Non-overlapping optimized Schwarz method, Ventell transmission condition, DDFV finite volume scheme, Anistropic diffusion.

Received : February 16, 2020 - Accepted: November 25, 2020.

(C)The Author(s) 2020. This article is published with open access by Sidi Mohamed Ben Abdallah University.

${ }^{1}$ University of Geneva, 2-4 rue du Lièvre, CP 64, 1211 Genève, Switzerland.

e-mail ${ }^{1}$ : martin.gander@unige.ch

${ }^{2}$ LAGA, Université Sorbonne Paris Nord et CNRS, 93430 Villetaneuse, FRANCE.

e-mail²: halpern@math.univ-paris13.fr (Corresponding Author).

${ }^{3}$ Aix Marseille Univ, CNRS, Centrale Marseille, I2M, Marseille, France, 39 rue F. Joliot Curie, 13453 Marseille, Cedex 13.

e-mail ${ }^{3}$ : florence.hubert@univ-amu.fr

${ }^{4}$ Université de Nice, Parc Valrose, 28 avenue Valrose, 06108 Nice, Cedex 2, FRANCE.

e-mail ${ }^{4}$ : krell@unice.fr . 


\section{Introduction}

Optimized Schwarz methods are a modern class of Schwarz methods which use instead of the classical Dirichlet transmission conditions at the interfaces more effective transmission conditions, which can take the physics of the problem at hand into account, see [18, 19] and references therein. This property is especially important for anisotropic diffusion problems, which behave very differently at interfaces depending on the orientation of the diffusion, see for example [24], [14, Section 5], and the very recent reference [35]; for classical Schwarz methods applied to anisotropic diffusion, see $[36,8,11]$, and for a specific earlier two level preconditioner [32]. Similarly when discretizing anisotropic diffusion problems, the numerical scheme must be suitable for high anisotropy, and discrete duality finite volume (DDFV) methods have this property, even in the case of discontinuous anisotropic diffusion, see [27, 5, 26, 6, 9, 15, 2], and in particular [16, Part II] which is dedicated especially to anisotropic diffusion. We are therefore interested in optimized Schwarz methods which are discretized using DDFV schemes. DDFV schemes belong to the class of discretization methods which preserve certain geometric properties of the underlying differential operators, like mimetic finite difference methods $[28,7]$, gradient methods [13], or discrete variational derivative methods [17], see also finite element exterior calculus [3]. DDFV methods are thus part of the effort to lead the field of geometric numerical integration, which reached a certain maturity for ordinary differential equations [25] to the area of partial differential equations.

Our paper is organized as follows: in Section 2, we present a class of non-overlapping optimized Schwarz methods for fully anisotropic diffusion at the continuous level, prove well posedness of the subdomain problems and give a convergence analysis using energy estimates for general Ventcell transmission conditions. We use an (arbitrary) two subdomain decomposition, but the generalization to the many subdomain case without cross points presents no difficulty $^{1}$. We then derive a convergence factor for the method, which is classically done for optimized Schwarz methods in the specific case of two unbounded or rectangular subdomains using Fourier analysis. We define the associated best approximation problem, and present a general theory for such problems which allows us to solve it, leading to our main results of closed form asymptotic formulas for the best choice of parameters of Robin and Ventcell type in Corollary 2.1 for unbounded domains, and in Theorem 2.8 for the case of bounded domains, where we used for the first time semi-asymptotic techniques. In Section 3 we then present a Discrete Duality Finite Volume discretization of the optimized Schwarz method, which naturally also allows the use of non-matching grids; note that this requires usually special techniques, see for example the cement method in the finite element case in [29, 30]. We prove well-posedness of the discrete subdomain problems, and convergence of the algorithm using discrete energy estimates for general Ventcell transmission conditions. For simplicity of notation, we show the results again for a two subdomain decomposition, but the generalization to the many subdomain case for decompositions without cross points is straightforward. In Section 4, we first test our new optimized Schwarz algorithms for anisotropic diffusion in

\footnotetext{
${ }^{1}$ Cross points need special treatment and are beyond the scope of the present analysis, see the conclusions for
} more information. 
the case covered by our analysis, i.e. on a rectangle decomposed into two rectangular subdomains, and investigate numerically for which kinds of anisotropic diffusion our new bounded domain analysis is important for the choice of the optimized parameters. We then also test the algorithm in situations not covered by the analysis, i.e. non-rectangular subdomains and for the many subdomain case. We present our conclusions and an outlook for further work in Section 5.

\section{Analysis at the continuous level}

We are interested in solving anisotropic diffusion problems of the form

$$
\begin{aligned}
\mathcal{L} u:=-\operatorname{div}(A \nabla u)+\eta u & =f & \text { in } \Omega, \\
u & =0 & \text { on } \partial \Omega,
\end{aligned}
$$

where $A$ is a symmetric positive definite matrix with $W^{1, \infty}$ coefficients,

$$
(x, y) \in \Omega \mapsto A(x, y)=\left(\begin{array}{ll}
A_{x x}(x, y) & A_{x y}(x, y) \\
A_{x y}(x, y) & A_{y y}(x, y)
\end{array}\right),
$$

and $(x, y) \in \Omega \mapsto \eta(x, y) \geq 0$ is a given non-negative function in $L^{\infty}(\Omega)$. To solve such problems on a computer, they have to be discretized, and we will use DDFV methods to do so in Section 3. Schwarz algorithms are however most naturally formulated and studied at the continuous level, and we will thus work first without discretization.

2.1. Schwarz Algorithm for anisotropic diffusion. For simplicity, we consider a decomposition of the domain $\Omega$ into two non-overlapping subdomains $\Omega_{j}, j=1,2$ with a vertical interface $\Gamma$. A parallel optimized Schwarz algorithm for the anisotropic diffusion problem (2.1) then solves for $\ell=1,2, \ldots$

$$
\begin{aligned}
\mathcal{L} u_{j}^{\ell} & =f & & \text { in } \Omega_{j}, \\
u_{j}^{\ell} & =0 & & \text { on } \partial \Omega_{j} \cap \partial \Omega_{,} \\
\left(A \nabla u_{j}^{\ell}, \mathbf{n}_{j}\right)+\Lambda u_{j}^{\ell} & =-\left(A \nabla u_{i}^{\ell-1}, \mathbf{n}_{i}\right)+\Lambda u_{i}^{\ell-1} & & \text { on } \Gamma=\partial \Omega_{1} \cap \partial \Omega_{2},
\end{aligned}
$$

where $j=1,2, i=2,1$, and $\mathbf{n}_{j}$ denotes the unit outer normal in $\Omega_{j}$. The transverse operator $\Lambda$ depends on two optimization coefficients $p$ and $q$, and is given by

$$
\Lambda u:=p u-q \partial_{y}\left(A_{y y} \partial_{y} u\right)
$$

which represents a so called Ventcell or second order transmission condition. The coefficient $p$ is strictly positive, since Ventcell conditions with $p=0$ would lead to less efficient transmission conditions. In the case $q=0$ we obtain a Robin transmission condition.

We first show that the subdomain problems of the form

$$
\begin{array}{rlrl}
\mathcal{L} u & =f & \text { in } \Omega, \\
u & =0 & \text { on } \partial \Omega_{D}, \\
(A \nabla u, \mathbf{n})+\Lambda u & =g & & \text { on } \partial \Omega_{V},
\end{array}
$$

which appear in algorithm (2.2) are well posed. An analysis of the Ventcell problem when $\partial \Omega=\partial \Omega_{V}$ is a regular curve in $\mathbb{R}^{2}$ can be found in [31]. 
Theorem 2.1 (Well-posedness of Subdomain Problems). Suppose $\Omega$ is convex, $A$ is in $W^{1, \infty}(\Omega)$, $\eta \geq 0$ in $L^{\infty}(\Omega)$ and $(A(\boldsymbol{x}) \boldsymbol{\xi}, \boldsymbol{\xi}) \geq \bar{A}\|\boldsymbol{\xi}\|^{2}>0$ for all $\boldsymbol{x}$ in $\Omega$ and $\boldsymbol{\xi} \in \mathbb{R}^{2}$ non zeros, and $p>0$ and $q \geq 0$. For any $(f, g) \in L^{2}(\Omega) \times L^{2}(\partial \Omega)$, problem (2.4) admits a unique solution $u$. If $q=0, u$ is in $H^{1}(\Omega)$, and if $q \neq 0, u$ is in

$$
\mathcal{W}(\Omega)=\left\{u \in H^{2}(\Omega), u=0 \text { on } \partial \Omega_{D}, \gamma_{\partial \Omega_{V}} u \in H^{2}\left(\partial \Omega_{V}\right) \cap H_{0}^{1}\left(\partial \Omega_{V}\right)\right\},
$$

where $\gamma \partial \Omega_{V}$ stands for the trace of $u$ on $\partial \Omega_{V}$.

Proof. The proof is based on a variational formulation in

$$
H_{1, \#}^{1}(\Omega):=\left\{u \in H^{1}(\Omega), u=0 \text { on } \Gamma^{D}, \gamma \partial \Omega_{V} u \in H_{0}^{1}\left(\partial \Omega_{V}\right)\right\},
$$

which is obtained by multiplying (2.4) by $v$ and integrating by parts. We introduce the bilinear forms

$$
\begin{aligned}
& a_{\Omega}(u, v):=\int_{\Omega} A \nabla u \nabla v d x d y+\int_{\Omega} \eta u v d x d y, \\
& a(u, v):=a_{\Omega}(u, v)+\langle\Lambda u, v\rangle_{\partial \Omega_{V^{\prime}}}
\end{aligned}
$$

where the last term must be understood as a duality product in $H_{0}^{1}(\Gamma)$, which can be rewritten in variational form as

$$
\langle\Lambda u, v\rangle_{\partial \Omega_{V}}=p \int_{\partial \Omega_{V}} u v d y+q \int_{\partial \Omega_{V}} A_{y y} \partial_{y} u \partial_{y} v d y
$$

$\Lambda$ is a self-adjoint continuous coercive operator from $H_{0}^{1}\left(\partial \Omega_{V}\right)$ onto $H^{-1}\left(\partial \Omega_{V}\right)$. It has a continuous self-adjoint inverse, defining a scalar product on $H^{-1}\left(\partial \Omega_{V}\right)$ by

$$
\langle u, v\rangle_{\Lambda^{-1}}:=\left\langle v, \Lambda^{-1} u\right\rangle_{\partial \Omega_{V}} .
$$

Computing

$$
a(u, u)=\int_{\Omega} A|\nabla u|^{2} d x d y+\int_{\Omega} \eta u^{2} d x d y+p \int_{\partial \Omega_{V}} u^{2} d y+q \int_{\partial \Omega_{V}} A_{y y}\left(\partial_{y} u\right)^{2} d y,
$$

we see that $a$ is a bilinear continuous coercive form on $H_{1, \#}^{1}(\Omega)$, equipped with the scalar product

$$
(w, v)_{H_{1, H}^{1}(\Omega)}=(\nabla w, \nabla v)_{L^{2}(\Omega)}+\left\langle\partial_{y} w, \partial_{y} v\right\rangle_{\partial \Omega_{V}}
$$

This gives existence and uniqueness of a weak solution in $H_{1, \#}^{1}(\Omega)$, i.e. a solution of

$$
a(u, v)=(f, v)_{L^{2}(\Omega)}+\langle g, v\rangle_{\partial \Omega_{V}} .
$$

If $q=0$, the proof holds in $H^{1}(\Omega)$.

For regularity results, $u$ is such that $\Delta u \in L^{2}(\Omega), \gamma \partial \Omega_{D} u=0$ and $\left(\partial_{x}-\partial_{y y}\right) \gamma \partial \Omega_{V} u \in L^{2}\left(\partial \Omega_{V}\right)$. Such a regularity result was proved in [37] for a regular boundary with Ventcell boundary condition all around, but due to the convexity of the domain, the result applies here. 
2.2. Convergence analysis using energy estimates. We now prove that the optimized Schwarz algorithm (2.2) converges when applied to the anisotropic diffusion problem (2.1).

Theorem 2.2 (Convergence of the Optimized Schwarz Algorithm). For any initial guess $\left(u_{1}^{0}, u_{2}^{0}\right)$ in $\mathcal{W}\left(\Omega_{1}\right) \times \mathcal{W}\left(\Omega_{2}\right)$, (2.2) defines a sequence of iterates in $\mathcal{W}\left(\Omega_{1}\right) \times \mathcal{W}\left(\Omega_{2}\right)$. If the solution $u$ of (2.1) is such that $\Lambda u \in L^{2}(\Gamma)$, the sequence converges in $H^{1}\left(\Omega_{1}\right) \times H^{1}\left(\Omega_{2}\right)$ to $u$.

Proof. The existence of the iterates is a consequence of Theorem 2.1. The solution $u$ of the boundary value problem (2.1) satisfies the continuity of the trace of $u$ and the flux $F:=A \nabla u \cdot n$ on the interface. Therefore by linearity, with the assumption stated in the theorem, only the convergence to the zero solution of the sequences of iterates with $f=0$ has to be proved. Defining the continuous flux $F_{j}^{\ell}:=-A \nabla u_{j}^{\ell} \cdot \boldsymbol{n}_{j}$, the transmission condition on $\Gamma$ takes the form

$$
-F_{j}^{\ell}+\Lambda u_{j}^{\ell}=F_{i}^{\ell-1}+\Lambda u_{i}^{\ell-1}
$$

Multiplying the PDE in (2.2) by $u_{j}^{\ell}$ and integrating yields

$$
a_{\Omega_{j}}\left(u_{j}^{\ell}, u_{j}^{\ell}\right)+R^{\ell, j}=0, \quad R^{\ell, j}:=\left\langle F_{j}^{\ell}, u_{j}^{\ell}\right\rangle_{\Gamma} .
$$

The essential step now in the proof is that, using the scalar product defined by $\Lambda^{-1}$ in (2.7), the boundary term $R^{\ell, j}$ can be rewritten as the difference of squares,

$$
R^{\ell, j}=\left\langle F_{j}^{\ell}, \Lambda^{-1} \Lambda u_{j}^{\ell}\right\rangle_{\Gamma}=\left\langle F_{j}^{\ell}, \Lambda u_{j}^{\ell}\right\rangle_{\Lambda^{-1}}=\frac{1}{4}\left\|F_{j}^{\ell}+\Lambda u_{j}^{\ell}\right\|_{\Lambda^{-1}}^{2}-\frac{1}{4}\left\|F_{j}^{\ell}-\Lambda u_{j}^{\ell}\right\|_{\Lambda^{-1}}^{2}
$$

Inserting this last expression into (2.10), we obtain

$$
a_{\Omega_{j}}\left(u_{j}^{\ell}, u_{j}^{\ell}\right)+\frac{1}{4}\left\|F_{j}^{\ell}+\Lambda u_{j}^{\ell}\right\|_{\Lambda^{-1}}^{2}=\frac{1}{4}\left\|F_{j}^{\ell}-\Lambda u_{j}^{\ell}\right\|_{\Lambda^{-1}}^{2} .
$$

We can now replace the right hand side using the transmission condition (2.9), and get

$$
a_{\Omega_{j}}\left(u_{j}^{\ell}, u_{j}^{\ell}\right)+\frac{1}{4}\left\|F_{j}^{\ell}+\Lambda u_{j}^{\ell}\right\|_{\Lambda^{-1}}^{2}=\frac{1}{4}\left\|F_{i}^{\ell-1}+\Lambda u_{i}^{\ell-1}\right\|_{\Lambda^{-1}}^{2} .
$$

Summing this equality over the subdomains and the iterations, we obtain for any $\ell_{\max } \geq 1$, due to the telescopic sum, that

$$
\sum_{\ell=1}^{\ell_{\max }} \sum_{j=1}^{2} a_{\Omega_{j}}\left(u_{j}^{\ell}, u_{j}^{\ell}\right)+\frac{1}{4} \sum_{j=1}^{2}\left\|F_{j}^{\ell_{\max }}+\Lambda u_{j}^{\ell_{\max }}\right\|_{\Lambda^{-1}}^{2}=\frac{1}{4} \sum_{j=1}^{2}\left\|F_{j}^{0}+\Lambda u_{j}^{0}\right\|_{\Lambda^{-1}}^{2} .
$$

Since the right hand side is a fixed quantity, the left hand side must remain bounded for all $\ell_{\max }$, and thus the iterates $u_{j}^{\ell}$ tend to zero in $H^{1}\left(\Omega_{j}\right)$ as $\ell$ goes to infinity.

2.3. Convergence factor at the continuous level. The convergence proof by energy estimates from Theorem 2.2 does not tell us anything about how to choose the parameters $p$ and $q$ to obtain fast convergence. In order to obtain such information, a technique in optimized Schwarz methods is to study the algorithm on specific, simpler domains with constant coefficients using Fourier techniques [18]. We therefore choose now the domain $\Omega:=(-L, L) \times \mathbb{R}$ with subdomains $\Omega_{1}:=(-L, 0) \times \mathbb{R}$ and $\Omega_{2}:=(0, L) \times \mathbb{R}$ and suppose that the matrix $A$ and function $\eta$ are constant. A Fourier transform in the $y$ direction with Fourier parameter $k$ of 
(2.1) then leads in the homogeneous case for the error we are interested in to solve the ordinary differential equation

$$
-A_{x x} \frac{\partial^{2} \hat{u}}{\partial x^{2}}-2 i k A_{x y} \frac{\partial \hat{u}}{\partial x}+\left(\eta+k^{2} A_{y y}\right) \hat{u}=0,
$$

where $\hat{u}=\hat{u}(x, k)$ corresponds to the Fourier transformed solution of (2.1) with $f=0$. The characteristic equation of (2.11) is

$$
A_{x x} r^{2}+2 i k A_{x y} r-\left(\eta+k^{2} A_{y y}\right)=0 .
$$

Since $A$ is positive definite, there are two complex anti-conjugate roots

$$
r_{ \pm}(k)=\frac{-i k A_{x y} \pm D(k)}{A_{x x}}, \quad D(k):=\sqrt{\eta A_{x x}+k^{2} \operatorname{det} A}>0 .
$$

The transmission conditions in (2.2) then take the form

$$
\begin{aligned}
\left(A_{x x} \partial_{x}+i k A_{x y}+\Lambda(i k)\right) \hat{u}_{1}^{\ell} & =\left(A_{x x} \partial_{x}+i k A_{x y}+\Lambda(i k)\right) \hat{u}_{2}^{\ell-1} \\
\left(-A_{x x} \partial_{x}-i k A_{x y}+\Lambda(i k)\right) \hat{u}_{2}^{\ell} & =\left(-A_{x x} \partial_{x}-i k A_{x y}+\Lambda(i k)\right) \hat{u}_{1}^{\ell-1} .
\end{aligned}
$$

In the case when the subdomains are half spaces, $L=+\infty$, in order for the subdomain solutions $u_{j}^{\ell}$ to be temperate distributions, we must have

$$
\hat{u}_{1}^{\ell}(x, k)=C_{1}^{\ell}(k) e^{r_{+}(k) x}, \quad \hat{u}_{2}^{\ell}(x, k)=C_{2}^{\ell}(k) e^{r_{-}(k) x},
$$

and the transmission conditions in (2.13) give

$$
\begin{aligned}
& (P(k)+D(k)) C_{1}^{\ell}(k)=(P(k)-D(k)) C_{2}^{\ell-1}(k), \\
& (P(k)+D(k)) C_{2}^{\ell}(k)=(P(k)-D(k)) C_{1}^{\ell-1}(k),
\end{aligned}
$$

with $P(k):=\Lambda(i k)$. The convergence factor in this case is therefore

$$
\rho(P, k):=\frac{P(k)-D(k)}{P(k)+D(k)}, \quad P(k)=p+q A_{y y} k^{2},
$$

characterizing the contraction of the functions $C_{j}^{\ell}(k)$ in the subdomain solutions (2.14),

$$
C_{j}^{\ell}(k)=(\rho(P(k), k))^{2} C_{j}^{\ell-2}(k)=(\rho(P(k), k))^{2\left\lfloor\frac{\ell}{2}\right\rfloor} C_{j}^{\bmod (\ell, 2)}(k) .
$$

The smaller the convergence factor, the faster the convergence of the algorithm.

Suppose now that the subdomains are strips, and $L>0$ is a fixed quantity. Then the subdomain solutions are of the form

$$
\begin{gathered}
\hat{u}_{1}^{\ell}(x, k)=C_{1}^{\ell}(k) e^{r_{+}(k)(L+x)}+D_{1}^{\ell}(k) e^{r_{-}(k)(L+x),} \\
\hat{u}_{2}^{\ell}(x, k)=C_{2}^{\ell}(k) e^{r_{-}(k)(-L+x)}+D_{2}^{\ell}(k) e^{r_{+}(k)(-L+x) .} .
\end{gathered}
$$

The outer boundary conditions for the error we consider here are $\hat{u}_{1}^{\ell}(-L, k)=0$ and $\hat{u}_{2}^{\ell}(L, k)=$ 0 , which implies that $D_{1}^{\ell}(k)=-C_{1}^{\ell}(k)$ and $D_{2}^{\ell}(k)=-C_{2}^{\ell}(k)$, and therefore

$$
\begin{gathered}
\hat{u}_{1}^{\ell}(x, k)=C_{1}^{\ell}(k)\left(e^{r_{+}(k)(L+x)}-e^{r_{-}(k)(L+x)}\right), \\
\hat{u}_{2}^{\ell}(x, k)=C_{2}^{\ell}(k)\left(e^{r_{-}(k)(-L+x)}-e^{r_{+}(k)(-L+x)}\right) .
\end{gathered}
$$


The transmission conditions in (2.13) then give

$$
\begin{aligned}
\left(e^{r_{+}(k) L}(P(k)\right. & \left.+D(k))-e^{r_{-}(k) L}(P(k)-D(k))\right) C_{1}^{\ell}(k) \\
= & \left(e^{-r_{-}(k) L}(P(k)-D(k))-e^{-r_{+}(k) L}(P(k)+D(k))\right) C_{2}^{\ell-1}(k), \\
\left(e^{-r_{-}(k) L}(P(k)\right. & \left.+D(k))-e^{-r_{+}(k) L}(P(k)-D(k))\right) C_{2}^{\ell}(k) \\
= & \left(e^{r_{+}(k) L}(P(k)-D(k))-e^{r_{-}(k) L}(P(k)+D(k))\right) C_{1}^{\ell-1}(k) .
\end{aligned}
$$

There are therefore two components forming the convergence factor, one from domain 1 to domain 2 and one from domain 2 to domain 1 ,

$$
\begin{aligned}
& \rho_{1 \rightarrow 2}=\frac{P(k)\left(e^{-r_{-}(k) L}-e^{-r_{+}(k) L}\right)-D(k)\left(e^{-r_{-}(k) L}+e^{-r_{+}(k) L}\right)}{P(k)\left(e^{r_{+}(k) L}-e^{r_{-}(k) L}\right)+D(k)\left(e^{r_{+}(k) L}+e^{r_{-}(k) L}\right)}, \\
& \rho_{2 \rightarrow 1}=\frac{P(k)\left(e^{r_{+}(k) L}-e^{r_{-}(k) L}\right)-D(k)\left(e^{r_{+}(k) L}+e^{r_{-}(k) L}\right)}{P(k)\left(e^{-r_{-}(k) L}-e^{-r_{+}(k) L}\right)+D(k)\left(e^{-r_{-}(k) L}+e^{-r_{+}(k) L}\right)} .
\end{aligned}
$$

Dividing their product by the factors multiplying $P(k)$, we obtain

$$
\rho_{1 \rightarrow 2} \rho_{2 \rightarrow 1}=\frac{P(k)-D(k) \frac{e^{-r_{-}(k) L}+e^{-r_{+}(k) L}}{e^{-r_{-}(k) L}-e^{-r_{+}(k) L}}}{P(k)+D(k) \frac{e^{r_{+}(k) L}+e^{r_{-}(k) L}}{e^{r_{+}(k) L}-e^{r_{-}(k) L}}} \frac{P(k)-D(k) \frac{e^{r_{+}(k) L}+e^{r_{-}(k) L}}{e^{r_{+}(k) L}-e^{r_{-}(k) L}}}{P(k)+D(k) \frac{e^{-r_{-}(k) L}+e^{-r_{+}(k) L}}{e^{-r_{-}(k) L}-e^{-r_{+}(k) L}} .}
$$

A direct calculation shows that

$$
\frac{e^{-r_{-}(k) L}+e^{-r_{+}(k) L}}{e^{-r_{-}(k) L}-e^{-r_{+}(k) L}}=\frac{e^{r_{+}(k) L}+e^{r_{-}(k) L}}{e^{r_{+}(k) L}-e^{r_{-}(k) L}}=\operatorname{coth}\left(\frac{L}{A_{x x}} D(k)\right),
$$

and we therefore obtain $\rho_{1 \rightarrow 2} \rho_{2 \rightarrow 1}=\rho_{b}^{2}$, with the convergence factor

$$
\rho_{b}(P, k)=\frac{P(k)-D(k) \operatorname{coth}\left(\frac{L}{A_{x x}} D(k)\right)}{P(k)+D(k) \operatorname{coth}\left(\frac{L}{A_{x x}} D(k)\right)} .
$$

We see that the convergence factor $\rho_{b}(P, k)$ for the bounded domain case is very similar to the convergence factor $\rho(P, k)$ in (2.15) for the unbounded domain case, and converges to it for fixed $k$ as $L$ goes to infinity.

We however also notice that both convergence factors tend to 1 for high frequencies for any fixed parameter choice $p$ and $q$, and convergence for high frequencies can thus be arbitrarily slow. Fortunately, in a discrete setting, only frequencies smaller than the largest eigenvalue of the discrete transverse operator $\partial_{y y}$ intervene. Therefore it is of great importance to find coefficients $p$ and $q$ which minimize the maximum of the convergence factor over a set of bounded frequencies, $k \in\left[k_{\min }, k_{\max }\right]$, that is to find parameters $p$ and $q$ which minimize the maximum norm of $|\rho(P(\cdot), \cdot)|$, a best approximation problem we study next. 
2.4. Best approximation problem and general results. Since $D(k)$ defined in $(2.12)$ and $P(k)$ depend on $k^{2}$ only, we define $\mu:=k^{2}$, and on the unbounded domain the function

$$
f_{\infty}(\mu):=D(k)=\sqrt{\mu \operatorname{det} A+\eta A_{x x}} .
$$

and the corresponding function on the bounded domain,

$$
f_{L}(\mu):=D(k) \operatorname{coth}\left(\frac{L}{A_{x x}} D(k)\right)=f_{\infty}(\mu) \operatorname{coth}\left(\frac{L}{A_{x x}} f_{\infty}(\mu)\right) .
$$

Then the corresponding convergence factors become with $Q(\mu):=p+q A_{y y} \mu$

$$
\rho(Q, \mu)=\frac{Q(\mu)-f_{\infty}(\mu)}{Q(\mu)+f_{\infty}(\mu)} \quad \text { and } \quad \rho_{L}(Q, \mu)=e^{2 i k \frac{A_{x y}}{A_{x x}} L} \frac{Q(\mu)-f_{L}(\mu)}{Q(\mu)+f_{L}(\mu)} .
$$

Let $M$ be a segment in $\mathbb{R}_{+}^{*}, M:=\left[\mu_{\text {min }}, \mu_{\text {max }}\right]=\left[k_{\text {min }}^{2}, k_{\text {max }}^{2}\right] . f_{\infty}$ and $f_{L}$ are positive functions on $M$, the term $e^{2 i k \frac{A_{x y}}{A x x} L}$ in $\rho_{b}(Q, \mu)$ has modulus equal to 1 , and can be omitted in what follows, giving rise to two real best approximation problems which are of the form:

$$
\begin{aligned}
& \text { for } F(Q, \mu):=\frac{Q(\mu)-f(\mu)}{Q(\mu)+f(\mu)} \text { and } G(Q):=\sup _{\mu \in M}|F(Q(\mu), \mu)|, \\
& \text { find } Q_{n}^{*} \in \mathbf{P}_{n} \text { such that } \delta_{n}^{*}:=G\left(Q_{n}^{*}\right)=\inf _{Q \in \mathbf{P}_{n}} G(Q),
\end{aligned}
$$

where $\mathbf{P}_{n}$ is the space of polynomials of degree smaller or equal to $n$.

Definition 2.1 (Alternating sequence). Let $Q \in \mathbf{P}_{n}$. An alternating sequence of length $m$ for $F(Q(\cdot), \cdot)$ is a sequence of points $\left(\mu_{1}<\cdots<\mu_{m}\right)$ in $M$ such that

$$
\left|F\left(Q\left(\mu_{i}\right), \mu_{i}\right)\right|=\|F(Q(\cdot), \cdot)\|_{\infty}, \quad F\left(Q\left(\mu_{i}\right), \mu_{i}\right)=-F\left(Q\left(\mu_{i+1}\right), \mu_{i+1}\right) .
$$

Theorem 2.3. Let $M$ be a segment in $\mathbb{R}, n \geq 0$, and $f$ be a continuous positive function on $M$. Then $\delta_{n}^{*}<1$, and problem (2.20) has a unique solution $Q_{n}^{*}$, for which $F\left(Q_{n}^{*}(\cdot), \cdot\right)$ has an alternating sequence of at least $n+2$ points.

Proof. Existence, uniqueness and the alternation property are a consequence of a more general analysis in $\mathbb{C}$, see [4].

Theorem 2.4 (Homographic De la Vallée Poussin). Let $M$ be a segment in $\mathbb{R}, n \geq 0$, and $f$ be a continuous positive function on $M$. Then any polynomial $Q$ for which $F(Q(\cdot), \cdot)$ has an alternating sequence $\mu_{1}<\cdots<\mu_{n+2}$ of length $n+2$, and $\delta=\|F(Q(\cdot), \cdot)\|_{\infty}<1$, is the global minimum point $Q_{n}^{*}$ of $G$.

Proof. By the uniqueness theorem it suffices to prove that $\delta=\delta_{n}^{*}$. The proof is then by contradiction: assuming that $\delta>\delta_{n}^{*}$, we write for each $i$

$$
\underbrace{F\left(Q\left(\mu_{i}\right), \mu_{i}\right)}_{ \pm \delta}-\underbrace{F\left(Q_{n}^{*}\left(\mu_{i}\right), \mu_{i}\right)}_{\mid \leq \delta_{n}^{*}<\delta}=2 \frac{f\left(\mu_{i}\right)\left(Q\left(\mu_{i}\right)-Q_{n}^{*}\left(\mu_{i}\right)\right)}{\left(f\left(\mu_{i}\right)+Q\left(\mu_{i}\right)\right)\left(f\left(\mu_{i}\right)+Q_{n}^{*}\left(\mu_{i}\right)\right)} .
$$

Note first that $\delta<1$ if and only if $Q$ is positive on the interval. The denominator in the right hand side is therefore positive. Since $f\left(\mu_{i}\right)>0$ and since the left-hand side has the sign of $F\left(Q\left(\mu_{i}\right), \mu_{i}\right), Q\left(\mu_{i}\right)-Q_{n}^{*}\left(\mu_{i}\right)$ has the sign of $F\left(Q\left(\mu_{i}\right), \mu_{i}\right)$. Thus the polynomial $Q-Q_{n}^{*}$ 
alternates in sign at the $n+2$ points $\mu_{i}$. It must therefore have at least $n+1$ roots, and being of degree $n$ it must vanish identically, which implies $Q=Q_{n}^{*}$ and $\delta=\delta_{n}^{*}$.

We now use the general results in Theorem 2.3 and 2.4 for the concrete case of $n=0$ and $n=1$, which correspond to the Robin and Ventcell transmission conditions in the optimized Schwarz method.

Theorem 2.5 (Solution for $n=0$ ). If $f$ is positive and monotonic, problem (2.20) for $n=0$ has $a$ unique solution $Q_{0}^{*}$. The alternation points of $F\left(Q_{0}^{*}, \cdot\right)$ are the endpoints of the interval, $\mu_{\min }$ and $\mu_{\max }$, and

$$
\begin{aligned}
& Q_{0}^{*}=\sqrt{f\left(\mu_{\max }\right) f\left(\mu_{\min }\right)}, \\
& \delta_{0}^{*}=\left|F\left(Q_{0}^{*}, \mu_{\text {min }}\right)\right|=\left|\frac{\sqrt{f\left(\mu_{\max }\right)}-\sqrt{f\left(\mu_{\min }\right)}}{\sqrt{f\left(\mu_{\max }\right)}+\sqrt{f\left(\mu_{\text {min }}\right)}}\right| .
\end{aligned}
$$

Proof. By Theorem 2.3, there is a unique solution $Q_{0}^{*}$, and it alternates at least twice. Since $\delta_{0}^{*}<1, Q_{0}^{*}$ is positive, and since $f$ is monotonic, $\mu \mapsto F(Q, \mu)$ is monotonic as well, and the extrema can only be at the endpoints. Alternation at those points, $F\left(Q, \mu_{\min }\right)=-F\left(Q, \mu_{\max }\right)$ is equivalent to $Q=\sqrt{f\left(\mu_{\min }\right) f\left(\mu_{\max }\right)}$. By uniqueness, we then obtain that $Q_{0}^{*}=Q$.

Theorem 2.6 (Solution for $n=1$ ). If $f$ is positive, problem (2.20) for $n=1$ has a unique solution $Q_{1}^{*}$. Furthermore, if $f$ is twice continuously differentiable, monotonic, with $f^{\prime \prime}$ of constant sign, there exists a unique $\bar{\mu} \in\left(\mu_{\min }, \mu_{\max }\right)$ solution of

$$
g(\bar{\mu}):=\frac{f(\bar{\mu})}{f^{\prime}(\bar{\mu})}-\bar{\mu}=s, \quad s:=\frac{\mu_{\max } f\left(\mu_{\min }\right)-\mu_{\min } f\left(\mu_{\max }\right)}{f\left(\mu_{\max }\right)-f\left(\mu_{\min }\right)},
$$

such that $F\left(Q_{1}^{*}, \cdot\right)$ alternates at $\mu_{\min }, \bar{\mu}$ and $\mu_{\max }$. If $Q_{1}^{*}(\mu)=p_{1}^{*}+\tilde{q}_{1}^{*} \mu$, then the coefficients are given by

$$
\tilde{q}_{1}^{*}=\sqrt{\frac{f\left(\mu_{\min }\right) f(\bar{\mu})}{\left(s+\mu_{\min }\right)(s+\bar{\mu})}}, \quad p_{1}^{*}=s \tilde{q}_{1}^{*},
$$

and we obtain $\delta_{1}^{*}=\left|F\left(Q_{1}^{*}, \mu_{\min }\right)\right|$.

Proof. The assumptions in the Theorem imply that $f$ is strictly monotonic, and hence $s$ is well defined, since the denominator can not vanish, and also $f^{\prime}$ never vanishes. By Theorem 2.3, there exists a unique polynomial $Q_{1}^{*}$ with at least three alternation points for $\mu \rightarrow$ $F(Q(\mu), \mu)$, with $Q(\mu)=p+\tilde{q} \mu$. The extremum points can only be endpoints, or $\bar{\mu}$ such that $\partial_{\mu} F(Q(\mu), \mu)=0$. Fixing $p$ and $\tilde{q}$, we compute

$$
\partial_{\mu} F(Q(\mu), \mu)=\frac{f(\mu) \tilde{q}-f^{\prime}(\mu)(p+\tilde{q} \mu)}{(Q(\mu)+f(\mu))^{2}}=\tilde{q} f^{\prime}(\mu) \frac{g(\mu)-s}{(Q(\mu)+f(\mu))^{2}} .
$$

Since $f^{\prime}$ never vanishes, $\partial_{\mu} F(Q(\mu), \mu)$ vanishes if and only if the numerator vanishes, which leads to the first equation in (2.23). Since

$$
g^{\prime}(\mu)=-\frac{f(\mu) f^{\prime \prime}(\mu)}{f^{\prime 2}(\mu)}
$$


under the assumptions of the theorem, $g$ is monotonic, and so is the numerator in (2.25) which shows that the derivative in $\mu$ vanishes at most once. Therefore the alternations are at $\left(\mu_{\min }, \bar{\mu}, \mu_{\max }\right)$, and the alternation property is expressed by

$$
\frac{Q\left(\mu_{\min }\right)-f\left(\mu_{\min }\right)}{Q\left(\mu_{\min }\right)+f\left(\mu_{\min }\right)}=\frac{Q\left(\mu_{\max }\right)-f\left(\mu_{\max }\right)}{Q\left(\mu_{\max }\right)+f\left(\mu_{\max }\right)}=-\frac{Q(\bar{\mu})-f(\bar{\mu})}{Q(\bar{\mu})+f(\bar{\mu})^{\prime}},
$$

where $\bar{\mu}$ is defined from $s$ by the first equation in (2.23). The system can be rewritten in the form

$$
\frac{Q\left(\mu_{\min }\right)}{f\left(\mu_{\min }\right)}=\frac{Q\left(\mu_{\max }\right)}{f\left(\mu_{\max }\right)}=\frac{f(\bar{\mu})}{Q(\bar{\mu})} \text {. }
$$

The first equality can be solved for $s$ in (2.23), and the second equality gives a relation between $\tilde{q}$ and $\bar{\mu}$,

$$
\tilde{q}^{2}\left(s+\mu_{\min }\right)(s+\bar{\mu})=f\left(\mu_{\min }\right) f(\bar{\mu}) .
$$

With $p_{1}^{*}, \tilde{q}_{1}^{*}$ and $\bar{\mu}$ defined in $(2.23,2.24)$, we thus have three alternations with $\| G\left(p_{1}^{*}+\right.$ $\left.\tilde{q}_{1}^{*} \mu\right) \|_{\infty}<1$, and hence by Theorem 2.4 , this is the unique solution.

2.5. Optimized parameters for the Schwarz methods. According to the definition of the convergence factor in (2.15), the analysis of the best approximation problem above applies in the case of an unbounded domain $\Omega$ with $\mu:=k^{2}, f(\mu):=D(k)$, and $Q(\mu)=p+\tilde{q} \mu=p+q A_{y y} \mu$.

Theorem 2.7 (Best parameters for unbounded $\Omega$ ). Let $D(k)=: \sqrt{\eta A_{x x}+k^{2} \operatorname{det} A}$. The coefficient leading to the best convergence for the Robin Schwarz algorithm on the unbounded domain $\Omega$ is

$$
p_{0}^{*, \infty}=\sqrt{D\left(k_{\max }\right) D\left(k_{\min }\right)},
$$

with associated optimized convergence factor

$$
\rho_{0}^{*, \infty}=\left|\frac{\sqrt{D\left(k_{\max }\right)}-\sqrt{D\left(k_{\min }\right)}}{\sqrt{D\left(k_{\max }\right)}+\sqrt{D\left(k_{\text {min }}\right)}}\right| .
$$

The coefficients leading to the best convergence for the Ventcell Schwarz algorithm are

$$
\begin{aligned}
& p_{1}^{*, \infty}=\sqrt{\operatorname{det} A} \frac{k_{\text {max }}^{2} D\left(k_{\text {min }}\right)-k_{\text {min }}^{2} D\left(k_{\max }\right)}{\sqrt{2\left(k_{\max }^{2}-k_{\min }^{2}\right)\left(D\left(k_{\max }\right)-D\left(k_{\min }\right)\right)}\left(D\left(k_{\min }\right) \mathrm{D}\left(k_{\max }\right)\right)^{\frac{1}{4}}}, \\
& q_{1}^{*, \infty}=\frac{\sqrt{\operatorname{det} A}}{A_{y y}} \frac{\sqrt{D\left(k_{\max }\right)-D\left(k_{\min }\right)}}{\sqrt{2\left(k_{\max }^{2}-k_{\text {min }}^{2}\right)}\left(D\left(k_{\min }\right) \mathrm{D}\left(k_{\max }\right)\right)^{\frac{1}{4}}},
\end{aligned}
$$

with the even smaller optimized convergence factor

$$
\rho_{1}^{*, \infty}=\left|\frac{p_{1}^{*, \infty}+q_{1}^{*, \infty} A_{y y} k_{\text {min }}^{2}-D\left(k_{\text {min }}\right)}{p_{1}^{*, \infty}+q_{1}^{*, \infty} A_{y y} k_{\text {min }}^{2}+D\left(k_{\text {min }}\right)}\right| .
$$

Proof. This result has already been obtained using a different analysis and a transformation in [14, Section 5], and has even been extended to the case of discontinuous coefficients. We use here Theorem 2.5 and 2.6 with $f=f_{\infty}$ to give a different proof. First, for the case of Robin conditions, it suffices to replace the definition of $f$ into (2.22) from Theorem 2.5 to get (2.27) 
and (2.28). For the Ventcell conditions, let $s_{\infty}$ be as defined in (2.23); then the solution of (2.23) can be obtained in closed form,

$$
\bar{\mu}_{\infty}=s_{\infty}-2 \frac{\eta A_{x x}}{\operatorname{det} A} .
$$

To obtain the coefficients, we compute first the terms in $q_{1}^{*}$ defined in (2.24),

$$
f_{\infty}\left(\bar{\mu}_{\infty}\right)=\sqrt{s_{\infty} \operatorname{det} A-\eta A_{x x}}, \quad \bar{\mu}_{\infty}+s_{\infty}=\frac{2}{\operatorname{det} A}\left(s_{\infty} \operatorname{det} A-\eta A_{x x}\right),
$$

and a direct computation shows that the common term can be expressed as

$$
s_{\infty} \operatorname{det} A-\eta A_{x x}=f_{\infty}\left(\mu_{\min }\right) f_{\infty}\left(\mu_{\max }\right) \text {. }
$$

Substituting this into the the terms in (2.31) and inserting them into the formula for $\tilde{q}_{1}^{*}$ defined in (2.24), we obtain $\tilde{q}_{1}^{*, \infty}$ in (2.29) when replacing $\mu$ by $k^{2}$ and $f_{\infty}$ by $D(k)$, and the corresponding expression for $q_{1}^{*, \infty}=\tilde{q}_{1}^{*, \infty} / A_{y y}$ in (2.29) follows. Then $p_{1}^{*, \infty}=s_{\infty} \tilde{q}_{1}^{*, \infty}$ from (2.24).

As mentioned earlier, the upper bound on the frequency $k_{\max }$ is related to the largest eigenvalue of the discrete transverse operator $\partial_{y y}$, that is $k_{\max } \sim \frac{\pi}{h}$, where $h$ is the mesh size along the interface [18], and we obtain the following asymptotic result.

Corollary 2.1 (Asymptotic performance on unbounded $\Omega$ ). For small mesh size $h$, i.e. large $k_{\max }=\frac{\pi}{h}$, the best Robin parameter and associated convergence factor behaves like

$$
\begin{aligned}
& p_{0}^{*, \infty}=\sqrt{D\left(k_{\text {min }}\right)} \sqrt[4]{\operatorname{det} A} k_{\text {max }}^{\frac{1}{2}}+\mathcal{O}\left(k_{\text {max }}^{-\frac{3}{2}}\right), \\
& \rho_{0}^{*, \infty}=1-2 \frac{\sqrt{D\left(k_{\text {min }}\right)}}{\sqrt[4]{\operatorname{det} A}} k_{\text {max }}^{-\frac{1}{2}}+\mathcal{O}\left(k_{\text {max }}^{-1}\right),
\end{aligned}
$$

and in the Ventcell case we obtain

$$
\begin{aligned}
p_{1}^{*, \infty} & =\frac{D\left(k_{\text {min }}\right)^{\frac{3}{4}}}{\sqrt{2} \operatorname{det} A^{\frac{3}{8}}} k_{\text {max }}^{\frac{1}{4}}+\mathcal{O}\left(k_{\text {max }}^{-\frac{3}{4}}\right), \\
q_{1}^{*, \infty} & =\frac{\operatorname{det} A^{\frac{5}{8}}}{\sqrt{2} A_{y y} D\left(k_{\text {min }}\right)^{\frac{1}{4}}} k_{\text {max }}^{-\frac{3}{4}}+\mathcal{O}\left(k_{\text {max }}^{-\frac{7}{4}}\right), \\
\rho_{1}^{*, \infty} & =1-2 \sqrt{2} D\left(k_{\text {min }}\right)^{\frac{1}{4}} \operatorname{det} A^{\frac{3}{8}} k_{\text {max }}^{-\frac{1}{4}}+\mathcal{O}\left(k_{\text {max }}^{-\frac{1}{2}}\right) .
\end{aligned}
$$

Proof. It suffices to insert $D\left(k_{\text {max }}\right)=k_{\text {max }} \sqrt{\operatorname{det} A}\left(1+\mathcal{O}\left(k_{\text {max }}^{-2}\right)\right)$ into the closed form solutions of Theorem 2.7 and to expand then for $k_{\text {max }}$ large.

In the case of a bounded domain, it is unfortunately not possible to solve (2.23) in closed form, and we first use an auxiliary asymptotic approximation of the coth term in $f_{L}$ to obtain a very good approximation of the coefficients, in closed form:

Theorem 2.8 (Semi-asymptotic performance on bounded $\Omega$ ). Let $f_{\infty}$ and $f_{L}$ be the functions defined in $(2.18,2.19)$. The best Robin parameter and associated convergence factor on a bounded domain of size $2 L$ are in the Robin case

$$
p_{0}^{*, L}=\sqrt{f_{L}\left(k_{\text {min }}^{2}\right) f_{L}\left(k_{\text {max }}^{2}\right)}, \quad \rho_{0}^{*, L}=\left|\frac{\sqrt{f_{L}\left(k_{\text {max }}^{2}\right)}-\sqrt{f_{L}\left(k_{\text {min }}^{2}\right)}}{\sqrt{f_{L}\left(k_{\text {max }}^{2}\right)}+\sqrt{f_{L}\left(k_{\text {min }}^{2}\right)}}\right| .
$$


In the Ventcell case, we obtain

$$
\begin{aligned}
& p_{1}^{*, L}=\frac{\sqrt{\operatorname{det} A}\left(k_{\text {max }}^{2} f_{L}\left(k_{\text {min }}^{2}\right)-k_{\text {min }}^{2} f_{\infty}\left(k_{\text {max }}^{2}\right)\right)}{\sqrt{2\left(k_{\text {max }}^{2}-k_{\text {min }}^{2}\right) \sqrt{f_{\infty}\left(k_{\text {max }}^{2}\right)\left(f_{\infty}\left(k_{\text {max }}^{2}\right) f_{L}\left(k_{\text {min }}^{2}\right)-f_{\infty}^{2}\left(k_{\text {min }}^{2}\right)\right)\left(f_{\infty}\left(k_{\text {max }}^{2}\right)-f_{L}\left(k_{\text {min }}^{2}\right)\right)}}} \\
& +\mathcal{O}\left(e^{-k_{\max }^{2}}\right), \\
& q_{1}^{*, L}=\frac{1}{A_{y y}} \frac{\sqrt{\operatorname{det} A}\left(f_{\infty}\left(k_{\max }^{2}\right)-f_{L}\left(k_{\min }^{2}\right)\right)^{\frac{3}{4}}}{\sqrt{2\left(k_{\max }^{2}-k_{\min }^{2}\right) \sqrt{f_{\infty}\left(k_{\max }^{2}\right)\left(f_{\infty}\left(k_{\max }^{2}\right) f_{L}\left(k_{\min }^{2}\right)-f_{\infty}^{2}\left(k_{\min }^{2}\right)\right)}}}+\mathcal{O}\left(e^{-k_{\max }^{2}}\right),
\end{aligned}
$$

with associated convergence factor

$$
\rho_{1}^{*, L}=\left|\frac{p_{1}^{*, L}+q_{1}^{*, L} A_{y y} k_{\min }^{2}-f_{L}\left(k_{\min }^{2}\right)}{p_{1}^{*, \infty}+q_{1}^{*, \infty} A_{y y} k_{\min }^{2}+f_{L}\left(k_{\min }^{2}\right)}\right| .
$$

Proof. In the case of a bounded domain $\Omega$, where $f_{L}(\mu)=f_{\infty}(\mu) \operatorname{coth}\left(\frac{L}{A_{x x}} f_{\infty}(\mu)\right)$, Theorem 2.5 still applies, since $f_{L}$ is positive and strictly increasing, and we easily obtain (2.34) for the Robin case. For the Ventcell case, to use Theorem 2.6, we need to solve equation (2.23) with $f$ replaced by $f_{L}$, i.e. with

$$
g_{L}(\mu):=\frac{f_{L}(\mu)}{f_{L}^{\prime}(\mu)}-\mu
$$

To see that equation (2.23) still has a unique solution, we compute

$$
f_{L}^{\prime \prime}(\mu)=\frac{a^{2}}{4 f_{L}^{3}(\mu)}\left(2 Y^{2} \cosh Y-\sinh ^{2} Y \cosh Y-Y \sinh Y\right), \quad Y=\frac{L}{A_{x x}} f_{\infty}(\mu) .
$$

A series expansion in $Y$ of the function in the parentheses gives

$$
\sum_{n \geq 1} a_{n} Y^{2 n}, \quad a_{n}=\frac{4 n(n-1)+\frac{1}{4}-\frac{3^{2 n}}{4}}{(2 n) !}
$$

and for $n \geq 1$, all coefficients $a_{n}$ are negative. Therefore $g_{L}$ is strictly increasing, and the equation $g_{L}(\mu)=s$ from (2.23) has a unique solution, which is however not available in closed form. We thus use exponential asymptotics for large $\mu_{\max }$ and fixed $L$,

$$
\begin{gathered}
f_{L}\left(\mu_{\max }\right)=f_{\infty}\left(\mu_{\max }\right)\left(1+\mathcal{O}\left(e^{-\mu_{\max }}\right)\right), \\
s_{L}=\frac{\mu_{\max } f_{L}\left(\mu_{\min }\right)-\mu_{\min } f_{\infty}\left(\mu_{\max }\right)}{f_{\infty}\left(\mu_{\max }\right)-f_{L}\left(\mu_{\min }\right)}+\mathcal{O}\left(e^{-\mu_{\max }}\right) .
\end{gathered}
$$

Then $s_{L}=\mathcal{O}\left(\mu_{\text {max }}^{\frac{1}{2}}\right)$, and since the function $g_{L}$ is increasing, we can see that the solution $\bar{\mu}_{L}$ of (2.23) with $g=g_{L}$ from (2.36) must increase at infinity as well. Furthermore for large $\mu$

$$
g_{L}(\mu)=\frac{f_{L}(\mu)}{f_{L}^{\prime}(\mu)}-\mu=\frac{f_{\infty}(\mu)}{f_{\infty}^{\prime}(\mu)}\left(1+\mathcal{O}\left(e^{-\mu}\right)\right)-\mu=\mu+2 \frac{\eta A_{x x}}{\operatorname{det} A}+\mathcal{O}\left(e^{-\mu}\right) .
$$

Therefore, we obtain instead of $\bar{\mu}_{\infty}$ from the unbounded domain case in (2.30)

$$
\bar{\mu}_{L}=s_{L}-2 \frac{\eta A_{x x}}{\operatorname{det} A}+\mathcal{O}\left(e^{-\mu_{\max }}\right)
$$




$$
=\frac{\mu_{\max } f_{L}\left(\mu_{\min }\right)-\mu_{\min } f_{\infty}\left(\mu_{\max }\right)}{f_{\infty}\left(\mu_{\max }\right)-f_{L}\left(\mu_{\min }\right)}-2 \frac{\eta A_{x x}}{\operatorname{det} A}+\mathcal{O}\left(e^{-\mu_{\max }}\right),
$$

and for the terms in $q_{1}^{*}$ we get instead of (2.31)

$$
f_{L}^{2}\left(\bar{\mu}_{L}\right)=f_{\infty}^{2}\left(\bar{\mu}_{L}\right)+\mathcal{O}\left(e^{-\mu_{\max }}\right)=s_{L} \operatorname{det} A-\eta A_{x x}+\mathcal{O}\left(e^{-\mu_{\max }}\right),
$$

and

$$
s_{L}+\bar{\mu}_{L}=\frac{2}{\operatorname{det} A}\left(s_{L} \operatorname{det} A-\eta A_{x x}\right)+\mathcal{O}\left(e^{-\mu_{\max }}\right) .
$$

We now simplify asymptotically the common term,

$$
s_{L} \operatorname{det} A-\eta A_{x x}=f_{\infty}\left(\mu_{\max }\right) \frac{f_{\infty}\left(\mu_{\max }\right) f_{L}\left(\mu_{\min }\right)-f_{\infty}^{2}\left(\mu_{\min }\right)}{f_{\infty}\left(\mu_{\max }\right)-f_{L}\left(\mu_{\min }\right)}+\mathcal{O}\left(e^{-\mu_{\max }}\right),
$$

Inserting these results into (2.24) and simplifying, we obtain (2.35).

Corollary 2.2 (Asymptotic performance on bounded $\Omega$ ). For small mesh size $h$, i.e. large $k_{\max }=$ $\frac{\pi}{h}$, the best Robin parameter and associated convergence factor behave like

$$
\begin{gathered}
p_{0}^{*, L}=\sqrt{k_{\text {max }}} \sqrt[4]{\operatorname{det} A} \sqrt{f_{L_{\text {min }}}}+\mathcal{O}\left(k_{\text {max }}^{-\frac{3}{2}}\right), \\
\rho_{0}^{*, L}=1-2 \frac{\sqrt{f_{L_{\text {min }}}}}{\sqrt[4]{\operatorname{det} A}} \frac{1}{\sqrt{k_{\text {max }}}}+\mathcal{O}\left(k_{\text {max }}^{-1}\right),
\end{gathered}
$$

where $f_{L_{\text {min }}}:=\sqrt{\Delta\left(k_{\text {min }}\right)} \operatorname{coth}\left(\frac{L}{A_{x x}} \sqrt{\Delta\left(k_{\text {min }}\right)}\right)$, and in the Ventcell case we obtain

$$
\begin{aligned}
& p_{1}^{*, L}=\frac{f_{L_{\text {min }}^{\frac{3}{4}}}^{\frac{3}{\sqrt{2}} \operatorname{det} A^{\frac{3}{8}}} k_{\text {max }}^{\frac{1}{4}}+\mathcal{O}\left(k_{\text {max }}^{-\frac{3}{4}}\right),}{q_{1}^{*, L}=\frac{\operatorname{det} A^{\frac{5}{8}}}{\sqrt{2} A_{y y} f_{L_{\text {min }}^{\frac{1}{4}}}} k_{\text {max }}^{-\frac{3}{4}}+\mathcal{O}\left(k_{\text {max }}^{-\frac{7}{4}}\right),} \\
& \rho_{1}^{*, L}=1-2 \sqrt{2} f_{L_{\text {min }}^{\frac{1}{4}}}^{\frac{4}{4}} \operatorname{det} A^{\frac{3}{8}} k_{\text {max }}^{-\frac{1}{4}}+\mathcal{O}\left(k_{\text {max }}^{-\frac{1}{2}}\right) .
\end{aligned}
$$

Proof. It suffices to use in (2.35) the approximation

$$
f_{\infty}\left(k_{\text {max }}^{2}\right)=k_{\text {max }} \sqrt{\operatorname{det} A}\left(1+\mathcal{O}\left(k_{\text {max }}^{-2}\right)\right) .
$$

From our asymptotic analyses in Corollary 2.1 for the unbounded domain case and Corollary 2.2 for the bounded domain case, we can see that with Robin transmission conditions, the convergence factor behaves like $1-\frac{C}{\sqrt[4]{\operatorname{det} A}} \sqrt{h}$, where $h$ is the mesh size along the interface, with a slightly different constant $C$ that has an extra dependence on the ratio $\frac{L}{A_{x x}}$ in the bounded domain case, and thus the ratio of the domain size compared to the diffusion in the $\mathrm{x}$-direction will influence the performance of the method on bounded domains. With Ventcell transmission conditions, the convergence factor behaves like $1-C \operatorname{det} A^{\frac{3}{8}} h^{\frac{1}{4}}$, again with 


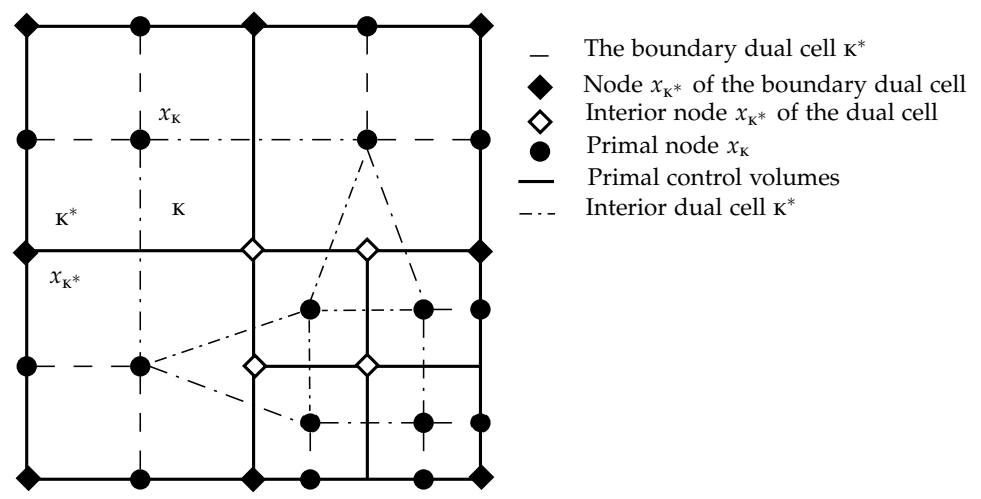

FIGURE 1. The mesh $\mathcal{T}$

a slightly different constant with the same extra dependence on the ratio $\frac{L}{A_{x x}}$ as in the Robin case. So Ventcell conditions will always lead to a much faster algorithm than Robin conditions, and a large or small ratio $\frac{L}{A_{x x}}$ will require the bounded domain analysis for accurate best parameter prediction.

\section{Analysis at the discrete level}

We now present the discrete duality finite volume discretization (DDFV) for anisotropic diffusion problems applied to the optimized Schwarz algorithms presented in Section 2 . The algorithm is built on the equation with variable diffusion matrix $A$.

3.1. Discrete duality finite volumes (DDFV). DDFV discretizations need a certain amount of notation for which we follow [1]. A DDFV mesh $\mathcal{T}$ consists of a primal mesh $\mathfrak{M}$ and a dual mesh $\mathfrak{M}^{*} \cup \partial \mathfrak{M}^{*}$, see Figure 1 for an illustration. The primal mesh $\mathfrak{M}$ is a set of disjoint open polygonal control volumes $\mathrm{K} \subset \Omega$ such that $\cup \overline{\mathrm{K}}=\bar{\Omega}$. We denote by $\partial \mathfrak{M}$ the set of edges of the control volumes in $\mathfrak{M}$ included in $\partial \Omega$, which we consider as degenerate control volumes. To each control volume and degenerate control volume $\mathrm{K} \in \mathfrak{M} \cup \partial \mathfrak{M}$, we associate a point $x_{\mathrm{K}} \in \mathrm{K}$. This family of points is denoted by $X=\left\{x_{\mathrm{K}}, \mathrm{K} \in \mathfrak{M} \cup \partial \mathfrak{M}\right\}$.

Let $X^{*}$ denote the set of the vertices $x_{\mathrm{K}^{*}}$ of the primal control volumes in $\mathfrak{M}$. We split this set into $X^{*}=X_{\text {int }}^{*} \cup X_{\text {ext }}^{*}$ where $X_{\text {int }}^{*} \cap \partial \Omega=\varnothing$ and $X_{\text {ext }}^{*} \subset \partial \Omega$. For all neighbor control volumes $\mathrm{K}$ and $\mathrm{L}$, we assume that $\partial \mathrm{K} \cap \partial \mathrm{L}$ is an edge of the primal mesh denoted by $\sigma=\mathrm{K} \mid \mathrm{L}$. We note by $\mathcal{E}$ the set of such edges.

Given the families of points $X$ and $X^{*}$, we define the diamond cells $\mathrm{D}$ to be the quadrangles whose diagonals are a primal edge $\sigma=\mathrm{K} \mid \mathrm{L}=\left(x_{\mathrm{K}^{*}}, x_{\mathrm{L}^{*}}\right)$ and the line $\left(x_{\mathrm{K}}, x_{\mathrm{L}}\right)$, see Figure 2 . We call the set of diamond cells $\mathfrak{D}$. A diamond cell $\mathrm{D}$ is an interior diamond cell, $\mathrm{D} \in \mathfrak{D}_{\text {int }}$, if $\left[x_{\mathrm{K}^{*}}, x_{\mathrm{L}^{*}}\right] \not \subset \partial \Omega$, and an exterior diamond cell $\mathrm{D} \in \mathfrak{D}_{\text {ext }}$ otherwise, and we have $\bar{\Omega}=\underset{\mathrm{D} \in \mathfrak{D}}{\cup} \overline{\mathrm{D}}$. To each diamond $\mathrm{D} \in \mathfrak{D}$, we associate a point $x_{\mathrm{D}} \in\left[x_{\mathrm{K}^{*}}, x_{\mathrm{L}^{*}}\right]$. Any interior diamond cell can thus be split into four triangles $\mathrm{D}=T_{\mathrm{KK}^{*}}^{\mathrm{D}} \cup T_{\mathrm{KL}^{*}}^{\mathrm{D}} \cup T_{\mathrm{LK}^{*}}^{\mathrm{D}} \cup T_{\mathrm{LL}^{*}}^{\mathrm{D}}$, and any exterior diamond cell into two triangles $\mathrm{D}=T_{\mathrm{KK}^{*}}^{\mathrm{D}} \cup T_{\mathrm{KL}^{*}}^{\mathrm{D}}$, see Figure 2. Let $\mathfrak{D}_{\mathrm{K}}$ be the set of diamonds with $x_{\mathrm{K}}$ as vertices 

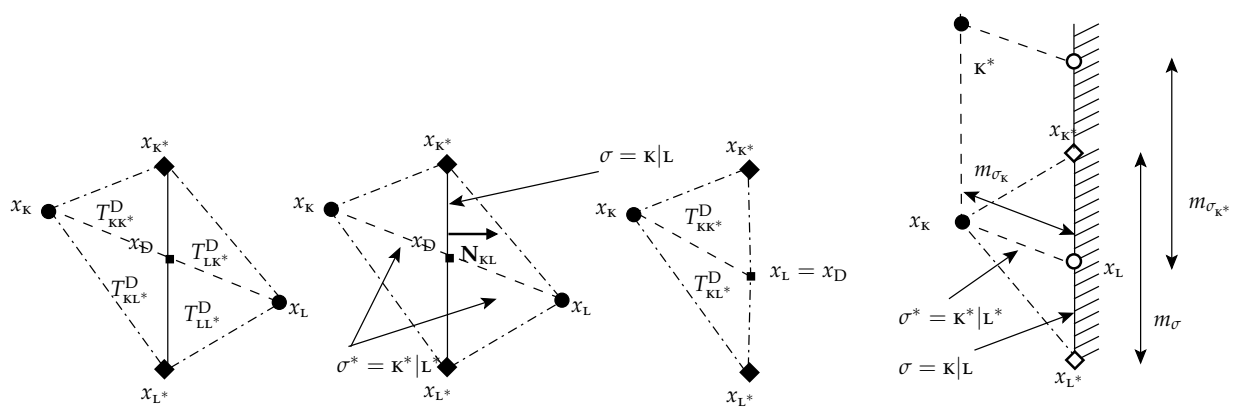

FIGURE 2. Notation in the diamond cells. Left: interior cell. Right: boundary dual and diamond cells.

and $\mathfrak{D}_{\mathrm{K}^{*}}$ the set of diamonds with $x_{\mathrm{K}^{*}}$ as vertices. We then observe that any primal cell can be described as

$$
\mathrm{K}=\cup_{\mathrm{D} \in \mathfrak{D}_{\mathrm{K}}}\left(T_{\mathrm{KK}^{*}}^{\mathrm{D}} \cup T_{\mathrm{KL}^{*}}^{\mathrm{D}}\right) \text {. }
$$

To any point $x_{\mathrm{K}^{*}} \in X^{*}$, we associate in a similar way the polygon $\mathrm{K}^{*}$ defined by

$$
\mathrm{K}^{*}:=\underset{\mathrm{D} \in \mathfrak{D}_{\mathrm{K}^{*}} \cap \mathcal{D}_{\text {int }}}{\cup}\left(T_{\mathrm{KK}^{*}}^{\mathrm{D}} \cup T_{\mathrm{LK}^{*}}^{\mathrm{D}}\right) \bigcup_{\mathrm{D} \in \mathfrak{D}_{\mathrm{K}^{*}} \cap \mathfrak{D}_{\text {ext }}}^{\cup} T_{\mathrm{KK}^{*}}^{\mathrm{D}} .
$$

This defines the set $\mathfrak{M}^{*} \cup \partial \mathfrak{M}^{*}$ of dual control volumes that forms a partition of $\Omega$ consisting of a family of disjoint polygonal control volumes. The dual edges are denoted by $\sigma^{*}=\mathrm{K}^{*} \mid \mathrm{L}^{*}$, and $\mathcal{E}^{*}$ is the set of dual edges.

For any primal control volume $\mathrm{K} \in \mathfrak{M} \cup \partial \mathfrak{M}$, we denote by $m_{\mathrm{K}}$ its Lebesgue measure, and the corresponding dual notation is $m_{\mathrm{K}^{*}}$. For a diamond cell $\mathrm{D}$ whose vertices are $\left(x_{\mathrm{K}}, x_{\mathrm{K}^{*}}, x_{\mathrm{L}}, x_{\mathrm{L}^{*}}\right)$, we denote by

- $m_{\mathrm{D}}$ its measure,

- $m_{\sigma}$ the length of the primal edge $\sigma$,

- $m_{\sigma^{*}}$ the length of the dual edge $\sigma^{*}$,

- $m_{\sigma_{\mathrm{K}^{*}}}$ the measure of $\partial \mathrm{K}^{*} \cap \Gamma$.

In DDFV, an unknown value $u_{\mathrm{K}}$ is associated with all primal control volumes $\mathrm{K} \in \mathfrak{M} \cup \partial \mathfrak{M}$, and an unknown value $u_{\mathrm{K}^{*}}$ is associated with all dual control volumes $\mathrm{K}^{*} \in \mathfrak{M}^{*} \cup \partial \mathfrak{M}^{*}$. We denote the approximate solution on the mesh $\mathcal{T}$ by $u_{\mathcal{T}} \in \mathbb{R}^{\mathcal{T}}$ where

$$
u_{\mathcal{T}}=\left(\left(u_{\mathrm{K}}\right)_{\mathrm{K} \in(\mathfrak{M} \cup \partial \mathfrak{M})},\left(u_{\mathrm{K}^{*}}\right)_{\mathrm{K}^{*} \in\left(\mathfrak{M}^{*} \cup \partial \mathfrak{M} *\right.}\right) .
$$

Following [10, 27, 12], we define a consistent approximation of the gradient operator denoted by $\nabla^{\mathfrak{D}}: u_{\mathcal{T}} \in \mathbb{R}^{\mathcal{T}} \mapsto\left(\nabla^{\mathrm{D}} u_{\mathcal{T}}\right)_{\mathrm{D} \in \mathfrak{D}} \in\left(\mathbb{R}^{2}\right)^{\mathfrak{D}}$,

$$
\nabla^{\mathrm{D}} u_{\mathcal{T}}:=\frac{1}{2 m_{\mathrm{D}}}\left[\left(u_{\mathrm{L}}-u_{\mathrm{K}}\right) \mathbf{N}_{\mathrm{KL}}+\left(u_{\mathrm{L}^{*}}-u_{\mathrm{K}^{*}}\right) \mathbf{N}_{\mathrm{K}^{*} \mathrm{~L}^{*}}\right], \quad \forall \mathrm{D} \in \mathfrak{D},
$$

with $\mathbf{N}_{\mathrm{KL}}=\left(x_{\mathrm{L}^{*}}-x_{\mathrm{K}^{*}}\right)^{\perp}$ and $\mathbf{N}_{\mathrm{K}^{*} \mathrm{~L}^{*}}=\left(x_{\mathrm{L}}-x_{\mathrm{K}}\right)^{\perp}$ with the convention $\left(\left(x_{\mathrm{L}^{*}}-x_{\mathrm{K}^{*}}\right) \wedge\left(x_{\mathrm{L}}-\right.\right.$ $\left.\left.x_{\mathrm{K}}\right), e_{z}\right)>0$. 
As in $[27,12]$, we also define a consistent approximation of the divergence operator denoted by $\operatorname{div}^{\mathcal{T}}: \xi_{\mathfrak{D}}=\left(\xi_{\mathrm{D}}\right)_{\mathrm{D} \in \mathfrak{D}} \mapsto \operatorname{div}^{\mathcal{T}} \xi_{\mathfrak{D}} \in \mathbb{R}^{\mathcal{T}}$ with

$$
\begin{gathered}
\operatorname{div}^{\mathrm{K}} \xi_{\mathfrak{D}}:=\frac{1}{m_{\mathrm{K}}} \sum_{\mathrm{D} \in \mathfrak{D}_{\mathrm{K}}}\left(\xi_{\mathrm{D}}, \mathbf{N}_{\mathrm{KL}}\right), \forall \mathrm{K} \in \mathfrak{M}, \\
\operatorname{div}^{\mathrm{K}^{*}} \xi_{\mathfrak{D}}:=\frac{1}{m_{\mathrm{K}^{*}}} \sum_{\mathrm{D} \in \mathfrak{D}_{\mathrm{K}^{*}}}\left(\xi_{\mathrm{D}}, \mathbf{N}_{\mathrm{K}^{*} \mathrm{~L}^{*}}\right), \forall \mathrm{K}^{*} \in \mathfrak{M}^{*} .
\end{gathered}
$$

The DDFV approximation $u_{\mathcal{T}} \in \mathbb{R}^{\mathcal{T}}$ of (2.1) is then solution to the linear system

$$
\begin{gathered}
-\operatorname{div}^{\mathrm{K}}\left(A_{\mathfrak{D}} \nabla^{\mathfrak{D}} u_{\mathcal{T}}\right)+\eta_{\mathrm{K}} u_{\mathrm{K}}=f_{\mathrm{K}}, \quad \forall \mathrm{K} \in \mathfrak{M}, \\
-\operatorname{div}^{\mathrm{K}^{*}}\left(A_{\mathfrak{D}} \nabla^{\mathfrak{D}} u_{\mathcal{T}}\right)+\eta_{\mathrm{K}^{*}} u_{\mathrm{K}^{*}}=f_{\mathrm{K}^{*}}, \quad \forall \mathrm{K}^{*} \in \mathfrak{M}^{*}, \\
u_{\mathrm{K}}=0, \quad \forall \mathrm{K} \in \partial \mathfrak{M}, \quad u_{\mathrm{K}^{*}}=0, \quad \forall \mathrm{K}^{*} \in \partial \mathfrak{M}^{*} .
\end{gathered}
$$

where

$$
\begin{aligned}
A_{\mathfrak{D}} & =\left(A_{\mathrm{D}}\right)_{\mathrm{D} \in \mathfrak{D}}, A_{\mathrm{D}}=A\left(x_{\mathrm{D}}\right), \\
f_{\mathcal{T}} & =\left(\left(f_{\mathrm{K}}\right)_{\mathrm{K} \in(\mathfrak{M} \cup \partial \mathfrak{M})},\left(f_{\mathrm{K}^{*}}\right)_{\mathrm{K}^{*} \in\left(\mathfrak{M}^{*} \cup \partial \mathfrak{M}^{*}\right)}\right), f_{\mathrm{K}}=f\left(x_{\mathrm{K}}\right), f_{\mathrm{K}^{*}}=f\left(x_{\mathrm{K}^{*}}\right), \\
\eta_{\mathcal{T}} & =\left(\left(\eta_{\mathrm{K}}\right)_{\mathrm{K} \in(\mathfrak{M} \cup \partial \mathfrak{M})},\left(\eta_{\mathrm{K}^{*}}\right)_{\mathrm{K}^{*} \in\left(\mathfrak{M}^{*} \cup \partial \mathfrak{M}^{*}\right)}\right), \eta_{\mathrm{K}}=\eta\left(x_{\mathrm{K}}\right), \eta_{\mathrm{K}^{*}}=\eta\left(x_{\mathrm{K}^{*}}\right)
\end{aligned}
$$

in case of smooth functions $A, f$ and $\eta$. Otherwise, mean values of the functions can be used.

3.2. DDFV on composite meshes. In the case of a domain decomposition into two subdomains $\Omega=\Omega_{1} \cup \Omega_{2}$, we need to consider for each subdomain $\Omega_{j}$ of $\Omega, j=1,2$, a DDFV mesh $\mathcal{T}_{j}=\left(\mathfrak{M}_{j} \cup \partial \mathfrak{M}_{j}, \mathfrak{M}_{j}^{*} \cup \partial \mathfrak{M}_{j}^{*}\right)$, and the associated diamond mesh $\mathfrak{D}_{j}$. Letting $\Gamma$ be the interface between $\Omega_{1}$ and $\Omega_{2}$, we denote by

$\mathfrak{D}_{j, \Gamma}:=\left\{\mathrm{D} \in \mathfrak{D}_{j}, \mathrm{D} \cap \Gamma \neq \varnothing\right\}$ the diamond cells intersecting $\Gamma$,

$\partial \mathfrak{M}_{j, \Gamma}:=\left\{\mathrm{K} \in \partial \mathfrak{M}_{j}, \mathrm{~K} \cap \Gamma \neq \varnothing\right\}$ the boundary primal cells intersecting $\Gamma$,

$\partial \mathfrak{M}_{j, \Gamma}^{*}:=\left\{\mathbf{K}^{*} \in \partial \mathfrak{M}_{j}^{*}, \mathbf{K}^{*} \cap \Gamma \neq \varnothing\right\}$ the boundary dual cells intersecting $\Gamma$,

$\partial \mathfrak{M}_{j, D}:=\left\{\mathrm{K} \in \partial \mathfrak{M}_{j}, \mathrm{~K} \cap \partial \Omega \neq \varnothing\right\}$ the boundary primal cells intersecting $\partial \Omega$,

$\partial \mathfrak{M}_{j, D}^{*}:=\left\{\mathrm{K}^{*} \in \partial \mathfrak{M}_{j}^{*}, \mathrm{~K}^{*} \cap \partial \Omega \neq \varnothing\right\}$ the boundary dual cells intersecting $\partial \Omega$.

For an example, see Figures 3 and 4. We will assume that the two meshes are compatible in the following sense:

(1) The two meshes have the same vertices on $\Gamma$. This implies in particular that the two meshes have the same degenerate control volumes on $\Gamma$, that is $\partial \mathfrak{M}_{1, \Gamma}=\partial \mathfrak{M}_{2, \Gamma}$. Let $N$ be the number of edges on $\Gamma$. For the sake of clarity, we sort these edges $\sigma_{1}, \cdots, \sigma_{N}$ in

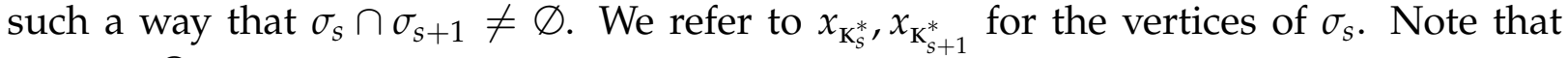
$x_{\mathrm{K}_{s}^{*}}=\sigma_{s} \cap \sigma_{s-1}$.

(2) The edges $\sigma_{S}$, whose center is denoted by $x_{\mathrm{L}_{s}}$, can be assimilated to a primal degenerated boundary control volume for both meshes, i.e. $\mathrm{L}_{s} \subset \partial \mathfrak{M}_{1} \cap \partial \mathfrak{M}_{2}$. 

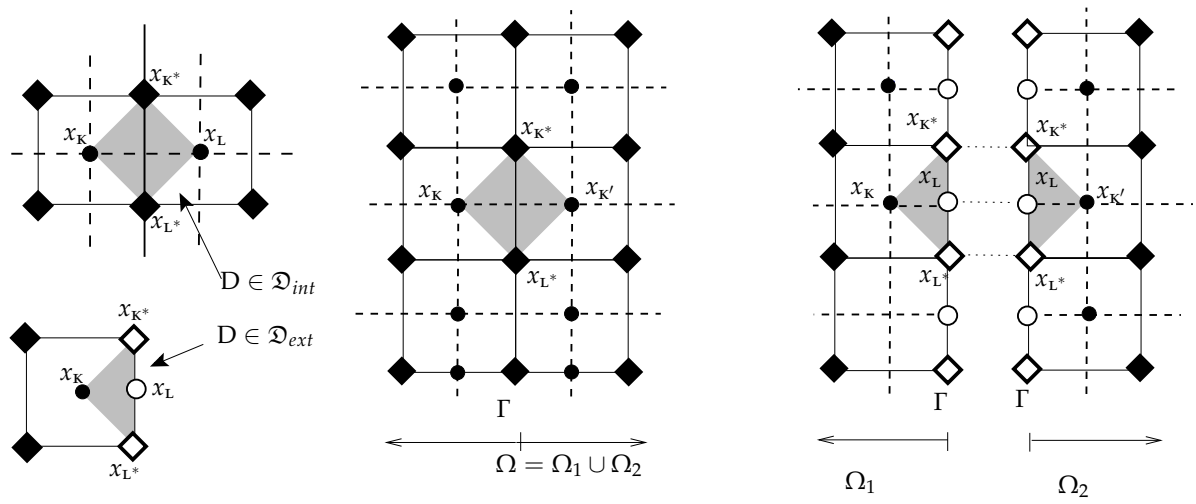

Figure 3. From a DDFV mesh $\mathcal{T}$ of the whole domain $\Omega$ to the $\mathcal{T}_{j}$ on $\Omega_{j}$.
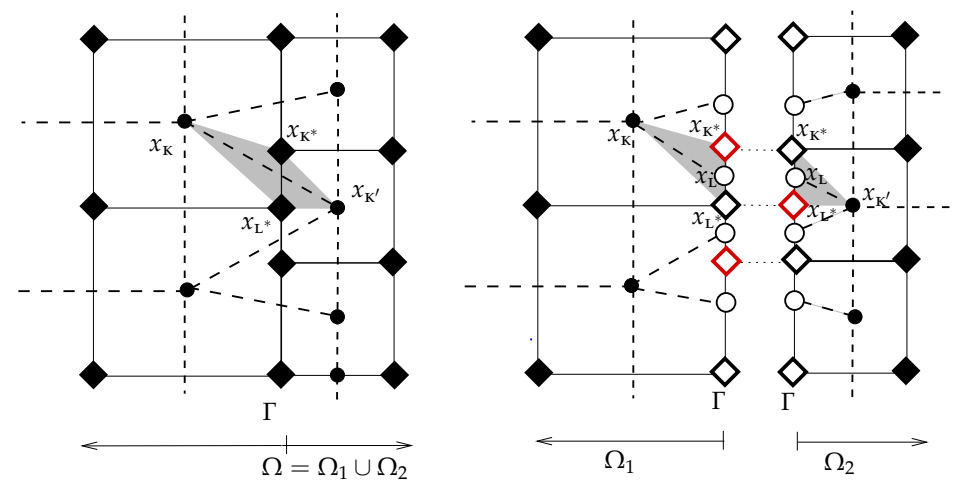

Figure 4. From a Nonconforming DDFV mesh $\mathcal{T}$ of the whole domain $\Omega$ to the $\mathcal{T}_{j}$ on $\Omega_{j}$.

Remark 3.1 (Non conforming meshes). In the definition of general finite volume meshes, the intersections of two neighboring control volumes are called edges of the mesh. Figure 4 (left) shows in the case of a non conforming mesh that a square volume may have more than 4 vertices. To create a compatible composite mesh from two a priori non conformal meshes, it is thus natural to add additional vertices on $\Gamma$ as shown in Figure 4 (right) in such a way that edges of $\partial \mathfrak{M}_{1, \Gamma}$ coincide with edges of $\partial \mathfrak{M}_{2, \Gamma}$.

We next define the DDFV discretization for the transmission conditions of Ventcell type. We associate one unknown per interior and exterior primal and dual cell $u_{\mathcal{T}_{j}} \in \mathbb{R}^{\mathcal{T}_{j}}$ and one flux unknown $\psi_{\mathrm{K}^{*}}$ for $j=1,2$, per interface dual cell $\mathrm{K}^{*} \in \partial \mathfrak{M}_{j, \Gamma}^{*}$. We denote by $\psi_{\mathcal{T}_{j}} \in \mathbb{R}^{\partial \mathfrak{M}_{j, \Gamma}^{*}}$ the collection of all flux unknowns $\psi_{\mathrm{K}^{*}}$, see Figure 5. For $u_{\mathcal{T}_{j}} \in \mathbb{R}^{\mathcal{T}_{j}}, \psi_{\mathcal{T}_{j}} \in \mathbb{R}^{\partial \mathfrak{M}_{j, \Gamma}^{*}}, f_{\mathcal{T}_{j}} \in$ $\mathbb{R}^{\mathfrak{M}_{j} \cup \mathfrak{M}_{j}^{*} \cup \partial \mathfrak{M}_{j}^{*}}$ and $h_{\mathcal{T}_{j}} \in \mathbb{R}^{\partial \mathfrak{M}_{j, \Gamma} \cup \partial \mathfrak{M}_{j, \Gamma}^{*}}$, we refer by

$$
\mathcal{L}_{\Omega_{j}, \Gamma}^{\mathcal{T}_{j}}\left(u_{\mathcal{T}_{j}}, \psi_{\mathcal{T}_{j}}, f_{\mathcal{T}_{j}}, h_{\mathcal{T}_{j}}\right)=0
$$

to the linear system

$$
-\operatorname{div}^{\mathrm{K}}\left(A_{\mathcal{D}} \nabla^{\mathfrak{D}} u_{\mathcal{T}_{j}}\right)+\eta_{\mathrm{K}} u_{\mathrm{K}}=f_{\mathrm{K}}, \quad \forall \mathrm{K} \in \mathfrak{M}_{j}
$$


OPTIMIZED SCHWARZ METHODS FOR ANISOTROPIC DIFFUSION WITH DDFV

$$
\begin{aligned}
& -\operatorname{div}^{\mathrm{K}^{*}}\left(A_{\mathfrak{D}} \nabla^{\mathcal{D}} u_{\mathcal{T}_{j}}\right)+\eta_{\mathrm{K}^{*}} u_{\mathrm{K}^{*}}=f_{\mathrm{K}^{*}}, \quad \forall \mathrm{K}^{*} \in \mathfrak{M}_{j}^{*}, \\
& -\frac{1}{m_{\mathrm{K}^{*}}}\left(\sum_{\mathrm{D} \in \mathfrak{D}_{\mathrm{K}^{*}}}\left(A_{\mathrm{D}} \nabla^{\mathrm{D}} u_{\mathcal{T}_{j}}, \mathbf{N}_{\mathrm{K}^{*} \mathrm{~L}^{*}}\right)+m_{\sigma_{\mathrm{K}^{*}}} \psi_{\mathrm{K}^{*}}\right)+\eta_{\mathrm{K}^{*}} u_{\mathrm{K}^{*}} \quad=\quad f_{\mathrm{K}^{*}}, \quad \forall \quad \mathrm{K}^{*} \in \partial \mathfrak{M}_{j, \Gamma}^{*}, \\
& \left(A_{\mathrm{D}} \nabla^{\mathrm{D}} u_{\mathcal{T}_{j}}, \mathbf{N}_{\mathrm{KL}}\right)+m_{\sigma} \Lambda_{\mathrm{L}}^{\partial \mathfrak{M}_{j, \Gamma}}\left(u_{\partial \mathfrak{M}_{j, \Gamma}}\right)=m_{\sigma} h_{\mathrm{L}}, \quad \forall \mathrm{L} \in \partial \mathfrak{M}_{j, \Gamma}, \\
& \psi_{\mathrm{K}^{*}}+\Lambda_{\mathrm{K}^{*}}^{\partial \mathfrak{M}_{j, \Gamma}^{*}}\left(u_{\partial \mathfrak{M}_{j, \Gamma}^{*}}\right)=h_{\mathrm{K}^{*}}, \quad \forall \mathrm{K}^{*} \in \partial \mathfrak{M}_{j, \Gamma}^{*}, \\
& u_{\mathrm{K}}=0, \quad \forall \mathrm{K} \in \partial \mathfrak{M}_{j, D}, \quad u_{\mathrm{K}^{*}}=0, \quad \forall \mathrm{K}^{*} \in \partial \mathfrak{M}_{j, D}^{*},
\end{aligned}
$$

with for $s=1, \cdots, N$

$$
\Lambda_{\mathrm{L}_{s}}^{\partial \mathfrak{M}_{j, \Gamma}}\left(u_{\partial \mathfrak{M}_{j, \Gamma}}\right)=p u_{\mathrm{L}_{s}}-\frac{q}{m_{\sigma_{s}}}\left(A_{y y}^{s^{*}+1} \frac{u_{\mathrm{L}_{s+1}}-u_{\mathrm{L}_{s}}}{m_{\sigma_{\mathrm{K}_{s}^{*}}}}-A_{y y}^{s^{*}} \frac{u_{\mathrm{L}_{s}}-u_{\mathrm{L}_{s-1}}}{m_{\sigma_{\mathrm{K}_{s}^{*}}}}\right)
$$

and for $s=2, \ldots, N$

$$
\Lambda_{\mathrm{K}_{s}^{*}}^{\partial \mathfrak{M}_{j, \Gamma}^{*}}\left(u_{\partial \mathfrak{M}_{j, \Gamma}^{*}}\right)=p^{*} u_{\mathrm{K}_{s}^{*}}-\frac{q^{*}}{m_{\sigma_{\mathrm{K}^{*}}}}\left(A_{y y}^{s} \frac{u_{\mathrm{K}_{s+1}^{*}}-u_{\mathrm{K}_{s}^{*}}}{m_{\sigma_{s}}}-A_{y y}^{s-1} \frac{u_{\mathrm{K}_{s}^{*}}-u_{\mathrm{K}_{s-1}^{*}}}{m_{\sigma_{s-1}}}\right) .
$$

where $A_{y y}^{s}$ and $A_{y y}^{s^{*}}$ are the values of $A_{y y}$ at the points $x_{\mathrm{L}_{s}}$ and $x_{\mathrm{K}_{s}^{*}}$. Note that $u_{\mathrm{L}_{0}}=u_{\mathrm{L}_{N+1}}=0$ and $u_{\mathrm{K}_{1}^{*}}=u_{\mathrm{K}_{N+1}^{*}}=0$ because of the boundary condition on $\partial \Omega$.

Equations (3.5a)-(3.5c) correspond to an approximation of the equation after integration on $\mathfrak{M}_{j}, \mathfrak{M}_{j}^{*}$ and $\partial \mathfrak{M}_{j}^{*}$. Equations (3.5d) and (3.5e) are related to the Ventcell transmission conditions on $\partial \mathfrak{M}_{j, \Gamma}$ and $\partial \mathfrak{M}_{j, \Gamma}^{*}$. Finally, equation (3.5f) corresponds to the homogeneous Dirichlet boundary condition on $\partial \Omega$.

3.3. DDFV Schwarz Algorithm for anisotropic diffusion. We can now present the optimized Schwarz algorithm discretized by DDFV: for an arbitrary initial guess $h_{\mathcal{T}_{j}}^{0} \in \mathbb{R}^{\partial \mathfrak{M}_{j, \Gamma} \cup \partial \mathfrak{M}_{j, \Gamma}^{*}, j \in}$ $\{1,2\}$, the algorithm performs for iteration index $\ell=0,1,2, \ldots$ and $i, j \in\{1,2\}, j \neq i$ the two steps:

(1) Compute $\left(u_{\mathcal{T}_{j}}^{\ell+1}, \psi_{\mathcal{T}_{j}}^{\ell+1}\right) \in \mathbb{R}^{\mathcal{T}_{j}} \times \mathbb{R}^{\partial \mathfrak{M}_{j, \Gamma}^{*}}$ solution to

$$
\mathcal{L}_{\Omega_{j}, \Gamma}^{\mathcal{T}_{j}}\left(u_{\mathcal{T}_{j}}^{\ell+1}, \psi_{\mathcal{T}_{j}}^{\ell+1}, f_{\mathcal{T}_{j}}, h_{\mathcal{T}_{j}}^{\ell}\right)=0 .
$$

(2) Compute the new values of $h_{\mathcal{T}_{j}}^{\ell+1}$ by

$$
\begin{gathered}
h_{\mathrm{L}}^{\ell+1}=-\frac{1}{m_{\sigma}}\left(A_{\mathrm{D}} \nabla^{\mathrm{D}} u_{\mathcal{T}_{i}}^{\ell+1}, \mathbf{N}_{\mathrm{KL}}\right)+\Lambda_{\mathrm{L}}^{\partial \mathfrak{M}_{i, \Gamma}}\left(u_{\partial \mathfrak{M}_{i, \Gamma}^{\ell+1}}\right), \forall \mathrm{L} \in \partial \mathfrak{M}_{j, \Gamma}, \\
h_{\mathrm{K}^{*}}^{\ell+1}=-\psi_{\mathrm{L}^{*}}^{\ell+1}+\Lambda_{\mathrm{L}^{*}}^{\partial \mathfrak{M}_{i, \Gamma}^{*}}\left(u_{\partial \mathfrak{M}_{i, \Gamma}^{\ell}}^{\ell+1}\right), \forall \mathrm{K}^{*} \in \partial \mathfrak{M}_{j, \Gamma}^{*} \text { and } \mathrm{L}^{*} \in \partial \mathfrak{M}_{i, \Gamma}^{*} \text { s. t. } x_{\mathrm{K}^{*}}=x_{\mathrm{L}^{*}} .
\end{gathered}
$$

To prove that this algorithm is well posed, we will need the following two Lemmas: 
Lemma 3.1 (Properties of $\Lambda^{\partial \mathfrak{M}_{j, \Gamma}}$ and $\Lambda^{\partial \mathfrak{M}_{j, \Gamma}^{*}}$ ). The operators $\Lambda^{\partial \mathfrak{M}_{j, \Gamma}}$ and $\Lambda^{\partial \mathfrak{M}_{j, \Gamma}^{*}}$ are symmetric and positive definite. The operators $\Lambda^{\partial \mathfrak{M}_{j, \Gamma}^{-1}}$ and $\Lambda^{\partial \mathfrak{M}_{j, \Gamma}^{*}-1}$ are also symmetric and positive definite, and induce a norm.

Proof. Using the weighted product on $\partial_{\mathfrak{M}_{\Gamma}},\left(u_{\partial \mathfrak{M}_{\Gamma}}, v_{\partial \mathfrak{M}_{\Gamma}}\right)=\sum_{s=1}^{N} m_{\sigma_{s}} u_{\mathrm{L}_{s}} v_{\mathrm{L}_{\mathcal{S}}}$, we show using (3.6) that

$$
\begin{aligned}
\left(\Lambda^{\partial \mathfrak{M}_{j, \Gamma}}\left(u_{\partial \mathfrak{M}_{j, \Gamma}}\right), v_{\partial \mathfrak{M}_{j, \Gamma}}\right)= & p \sum_{s=1}^{N} m_{\sigma_{s}} u_{\mathrm{L}_{s}} v_{\mathrm{L}_{s}} \\
& +q \sum_{s=0}^{N} m_{\sigma_{\mathrm{K}_{s}^{*}+1}} A_{y y}^{s^{*}+1} \frac{u_{\mathrm{L}_{s+1}}-u_{\mathrm{L}_{s}}}{m_{\sigma_{\mathrm{K}^{*}+1}}} \frac{v_{\mathrm{L}_{s+1}}-v_{\mathrm{L}_{s}}}{m_{\sigma_{\mathrm{K}_{s}}}},
\end{aligned}
$$

and we see the symmetry between $u$ and $v$, and this also implies the other properties of $\Lambda^{\partial \mathfrak{M}_{j, \Gamma}}$ and $\Lambda^{\partial \mathfrak{M}_{j, \Gamma}-1}$. Similarly, one can also obtain the properties of $\Lambda^{\partial \mathfrak{M}_{j, \Gamma}^{*}}$ and $\Lambda^{\partial \mathfrak{M}_{j, \Gamma}^{*}-1}$.

Lemma 3.2 (Variational form of the DDFV scheme). The vector $\left(u_{\mathcal{T}_{j}}, \psi_{\mathcal{T}_{j}}\right) \in \mathbb{R}^{\mathcal{T}_{j}} \times R^{\partial \mathfrak{M}_{j, \Gamma} \cup \partial \mathfrak{M}_{j, \Gamma}^{*}}$ is a solution of the linear system

$$
\mathcal{L}_{\Omega_{j}, \Gamma}^{\mathcal{T}_{j}}\left(u_{\mathcal{T}_{j}}, \psi_{\mathcal{T}_{j}}, f_{\mathcal{T}_{j}}, h_{\mathcal{T}_{j}}\right)=0
$$

if and only if for all $v_{\mathcal{T}_{j}} \in \mathbb{R}^{\mathcal{T}_{j}}$ we have

$$
\begin{aligned}
& 2 \sum_{\mathrm{D} \in \mathfrak{D}_{j}} m_{\mathrm{D}}\left(A_{\mathrm{D}} \nabla^{\mathrm{D}}{u_{\mathcal{T}_{j}}} \nabla^{\mathrm{D}_{v_{\mathcal{T}_{j}}}}\right)+\left(\Lambda^{\partial \mathfrak{M}_{j, \Gamma}}\left(u_{\partial \mathfrak{M}_{j, \Gamma}}\right), v_{\partial \mathfrak{M}_{j, \Gamma}}\right)+\left(\Lambda^{\partial \mathfrak{M}_{j, \Gamma}^{*}}\left(u_{\partial \mathfrak{M}_{j, \Gamma}^{*}}\right), v_{\partial \mathfrak{M}_{j, \Gamma}^{*}}\right) \\
& +\sum_{\mathbf{K} \in \mathfrak{M}_{j}} m_{\mathrm{K}} \eta_{\mathrm{K}} u_{\mathrm{K}} v_{\mathrm{K}}+\sum_{\mathrm{K}^{*} \in \mathfrak{M}_{j}^{*} \cup \partial \mathfrak{M}_{j, \Gamma}^{*}} m_{\mathrm{K}^{*}} \eta_{\mathrm{K}^{*}} u_{\mathrm{K}^{*}} v_{\mathrm{K}^{*}} \\
& =\sum_{\mathbf{K} \in \mathfrak{M}_{j}} m_{\mathrm{K}} f_{\mathrm{K}} v_{\mathrm{K}}+\sum_{\mathrm{K}^{*} \in \mathfrak{M}_{j}^{*} \cup \partial \mathfrak{M}_{j, \Gamma}^{*}} m_{\mathrm{K}^{*}} f_{\mathrm{K}^{*}} v_{\mathrm{K}^{*}} \\
& +\sum_{\mathbf{L} \in \partial \mathfrak{M}_{j, \Gamma}} m_{\sigma} h_{\mathrm{L}} v_{\mathrm{L}}+\sum_{\mathrm{K}^{*} \in \partial \mathfrak{M}_{j, \Gamma}^{*}} m_{\sigma_{\mathrm{K}^{*}}} h_{\mathrm{K}^{*}} v_{\mathrm{K}^{*}} .
\end{aligned}
$$

Proof. We multiply equation (3.5a) by $m_{\mathrm{K}} v_{\mathrm{K}}$ and equations (3.5b)-(3.5c) by $m_{\mathrm{K}^{*}} v_{\mathrm{K}^{*}}$ and sum theses identities over all the control volumes in $\mathfrak{M}_{j}$ and $\mathfrak{M}_{j}^{*} \cup \partial \mathfrak{M}_{j}^{*}$. Reordering the different contributions over all diamond cells, we obtain

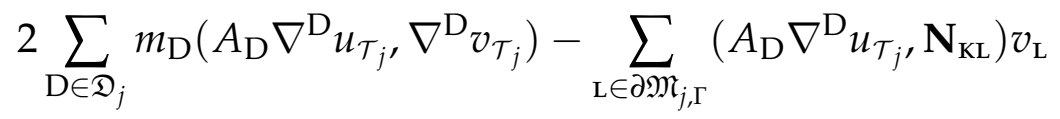

$$
\begin{aligned}
& -\sum_{\mathrm{K}^{*} \in \partial \mathfrak{M}_{j, \Gamma}^{*}} m_{\sigma_{\mathrm{K}^{*}}} \psi_{\mathrm{K}^{*}} v_{\mathrm{K}^{*}}+\sum_{\mathbf{K} \in \mathfrak{M}_{j}} m_{\mathrm{K}} \eta_{\mathrm{K}} u_{\mathrm{K}} v_{\mathrm{K}}+\sum_{\mathrm{K}^{*} \in \mathfrak{M}_{j}^{*} \cup \partial \mathfrak{M}_{j, \Gamma}^{*}} m_{\mathrm{K}^{*}} \eta_{\mathrm{K}^{*}} u_{\mathrm{K}^{*}} v_{\mathrm{K}^{*}} \\
& =\sum_{\mathbf{K} \in \mathfrak{M}_{j}} m_{\mathrm{K}} f_{\mathrm{K}} v_{\mathrm{K}}+\sum_{\mathrm{K}^{*} \in \mathfrak{M}_{j}^{*} \cup \partial \mathfrak{M}_{j, \Gamma}^{*}} m_{\mathrm{K}^{*}} f_{\mathrm{K}^{*}} v_{\mathrm{K}^{*}} .
\end{aligned}
$$

Introducing now the Ventcell transmission conditions (3.5d) and (3.5e), we obtain (3.10).

We can now prove that the subdomain problems discretized by DDFV are well posed: 

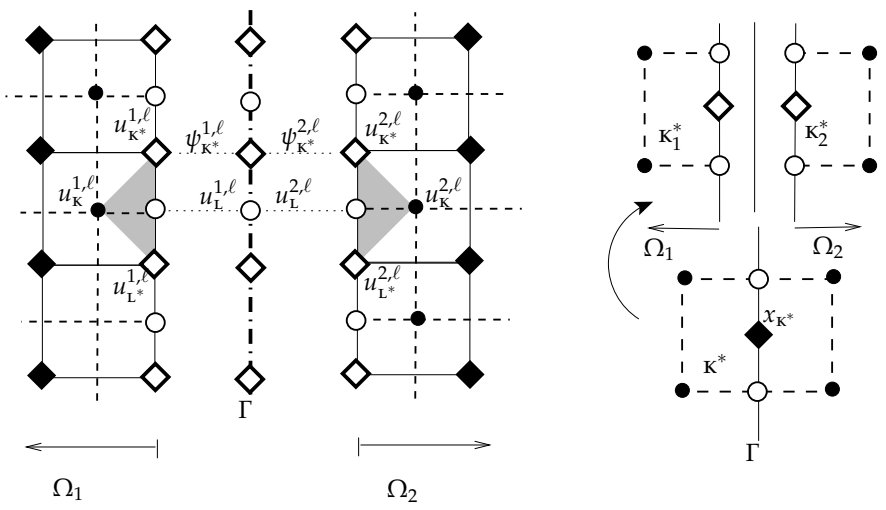

FIGURE 5. Notation around a diamond. The new unknowns needed to describe the DDFV scheme on $\Omega$ as the limit of the Schwarz algorithm.

Theorem 3.1 (Well-posedness of the DDFV Subdomain Problems). For any $f_{\mathcal{T}_{j}} \in \mathbb{R}^{\mathfrak{M}_{j} \cup \mathfrak{M}_{j}^{*} \cup \partial \mathfrak{M}_{j}^{*}}$

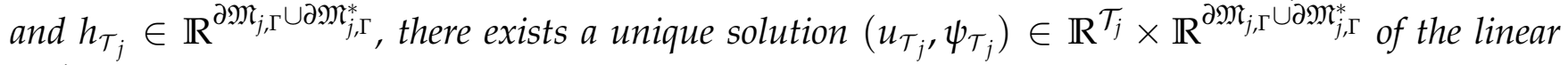
system

$$
\mathcal{L}_{\Omega_{j}, \Gamma}^{\mathcal{T}_{j}}\left(u_{\mathcal{T}_{j}}, \psi_{\mathcal{T}_{j}}, f_{\mathcal{T}_{j}}, h_{\mathcal{T}_{j}}\right)=0
$$

Proof. By linearity, it is sufficient to prove that if $\mathcal{L}_{\Omega_{j}, \Gamma}^{\mathcal{T}_{j}}\left(u_{\mathcal{T}_{j}}, \psi_{\mathcal{T}_{j}}, 0,0\right)=0$, then $u_{\mathcal{T}_{j}}=0$ and $\psi_{\mathcal{T}_{j}}=0$. We just use (3.10) with $v_{\mathcal{T}_{j}}=u_{\mathcal{T}_{j}}, f_{\mathcal{T}_{j}}=0, h_{\mathcal{T}_{j}}=0$ to obtain that

$$
\begin{aligned}
& 2 \sum_{\mathrm{D} \in \mathfrak{D}} m_{\mathrm{D}}\left(A_{\mathrm{D}} \nabla^{\mathrm{D}}{u_{\mathcal{T}_{j}}} \nabla^{\mathrm{D}}{u_{\mathcal{T}}}_{\mathcal{T}^{\prime}}\right)+\left(\Lambda^{\partial \mathfrak{M}_{j, \Gamma}}\left(u_{\partial \mathfrak{M}_{j, \Gamma}}\right), u_{\partial \mathfrak{M}_{j, \Gamma}}\right) \\
& +\left(\Lambda^{\partial \mathfrak{M}_{j, \Gamma}^{*}}\left(u_{\partial \mathfrak{M}_{j, \Gamma}^{*}}\right), u_{\partial \mathfrak{M}_{j, \Gamma}^{*}}\right)+\sum_{\mathbf{K} \in \mathfrak{M}_{j}} m_{\mathrm{K}} \eta_{\mathrm{K}} u_{\mathrm{K}}^{2}+\sum_{\mathrm{K}^{*} \in \mathfrak{M}_{j}^{*} \cup \partial \mathfrak{M}_{j, \Gamma}^{*}} m_{\mathrm{K}^{*}} \eta_{\mathrm{K}^{*}} u_{\mathrm{K}^{*}}{ }^{2}=0,
\end{aligned}
$$

which implies with Lemma 3.1 due to the non-negativity of all terms that both primal and dual unknowns are all identically zero.

3.4. DDFV convergence analysis using energy estimates. We now show how the technique of energy estimates we have used at the continuous level to prove convergence of the optimized Schwarz algorithm in Theorem 2.2 can be adapted to also prove convergence of the algorithm discretized by DDFV.

Theorem 3.2 (Convergence of the DDFV Schwarz algorithm). The iterates of the optimized Schwarz algorithm discretized by DDFV defined by (3.8)-(3.9) converge as $\ell$ tends to infinity to the solution $u_{\mathcal{T}}$ of the DDFV scheme (3.4) on $\Omega$.

Proof. We first rewrite the classical DDFV scheme (3.4) on $\Omega$ as the limit of the Schwarz algorithm. To this end, we introduce new unknowns near the boundary $\Gamma$, see Figure 5:

- for all $\mathrm{K} \in \mathfrak{M}_{j}$ and $\mathrm{K}^{*} \in \mathfrak{M}_{j}^{*}$, we set $u_{\mathrm{K}}^{\infty}=u_{\mathrm{K}}$ and $u_{\mathrm{K}^{*}}^{\infty}=u_{\mathrm{K}^{*}}$,

- for all $\mathrm{K} \in \partial \mathfrak{M}_{j, D}$ and $\mathrm{K}^{*} \in \partial \mathfrak{M}_{j, D}^{*}$, we set $u_{\mathrm{K}}^{\infty}=0$ and $u_{\mathrm{K}^{*}}^{\infty}=0$, 
- for all $\mathrm{L}_{j} \in \partial \mathfrak{M}_{j, \Gamma}$ and $\mathrm{L}_{i} \in \partial \mathfrak{M}_{i, \Gamma}$ such that $\mathrm{L}_{j}=\mathrm{L}_{i}$, we have $\mathrm{K}_{j} \in \mathfrak{M}_{j}$ such that $\mathrm{L}_{j} \in \partial \mathrm{K}_{j}$, we define $m_{\sigma_{\mathrm{K}_{j}}}$ to be the distance between $x_{\mathrm{K}_{j}}$ and $x_{\mathrm{D}}$. We also have $\mathrm{K}_{i} \in \mathfrak{M}_{i}$ such that $\mathrm{L}_{i} \in \partial \mathrm{K}_{i}$ and choose

$$
u_{\mathrm{L}_{j}}^{\infty}=u_{\mathrm{L}_{i}}^{\infty}=\frac{m_{\sigma_{\mathrm{K}_{i}}} u_{\mathrm{K}_{j}}+m_{\sigma_{\mathrm{K}_{j}}} u_{\mathrm{K}_{i}}}{m_{\sigma_{\mathrm{K}_{j}}}+m_{\sigma_{\mathrm{K}_{i}}}},
$$

in such a way that $\left(A_{\mathrm{D}_{j}} \nabla^{\mathrm{D}_{j}} u_{\mathcal{T}_{j}}^{\infty}, \mathbf{N}_{\mathrm{K}_{j} \mathrm{~L}}\right)=-\left(A_{\mathrm{D}_{i}} \nabla^{\mathrm{D}_{i}} u_{\mathcal{T}_{i}}^{\infty}, \mathbf{N}_{\mathrm{K}_{i} \mathrm{~L}}\right)$, with $\mathrm{D}_{j} \in \mathfrak{D}_{j, \Gamma}$ and $\mathrm{D}_{i, \Gamma} \in$ $\mathfrak{D}_{i}$ such that $x_{\mathrm{D}_{j}}=x_{\mathrm{L}_{j}}=x_{\mathrm{D}_{i}}=x_{\mathrm{L}_{i}}$.

- for all $\mathrm{K}^{*} \in \mathfrak{M}^{*}$ such that $\mathrm{K}^{*}=\mathrm{K}_{i}^{*} \cup \mathrm{K}_{j}^{*}$ with $\mathrm{K}_{j}^{*} \in \partial \mathfrak{M}_{j, \Gamma}^{*}$ and $\mathrm{K}_{i}^{*} \in \partial \mathfrak{M}_{i, \Gamma}^{*}$, choose $u_{\mathrm{K}_{j}^{*}}^{\infty}=u_{\mathrm{K}_{i}^{*}}^{\infty}=u_{\mathrm{K}^{*}}$ and

$$
\begin{aligned}
& \left.\psi_{\mathrm{K}_{j}^{*}}^{\infty}=-\psi_{\mathrm{K}_{i}^{*}}^{\infty}=-\frac{1}{m_{\sigma_{\mathrm{K}^{*}} \mathrm{D} \in \mathfrak{D}_{\mathrm{K}_{j}^{*}}}} \sum_{\mathrm{D}} \nabla^{\mathrm{D}}{u_{\mathcal{T}_{j}}^{\infty}}^{\infty} \mathbf{N}_{\mathrm{K}_{j}^{*} \mathrm{~L}_{j}^{*}}\right)+\frac{m_{\mathrm{K}_{j}^{*}}}{m_{\sigma_{\mathrm{K}^{*}}}}\left(\eta_{\mathrm{K}^{*}} u_{\mathrm{K}^{*}}-f_{\mathrm{K}^{*}}\right) \\
& \left.=\frac{1}{m_{\sigma_{\mathrm{K}^{*}} \mathrm{D} \in \mathfrak{D}_{\mathrm{K}_{i}^{*}}}} \sum_{A_{\mathrm{D}}} \nabla^{\mathrm{D}}{u_{\mathcal{T}_{i}}^{\infty}}^{\infty} \mathbf{N}_{\mathrm{K}_{i}^{*} \mathrm{~L}_{i}^{*}}\right)-\frac{m_{\mathrm{K}_{i}^{*}}}{m_{\sigma_{\mathrm{K}^{*}}}}\left(\eta_{\mathrm{K}^{*}} u_{\mathrm{K}^{*}}-f_{\mathrm{K}^{*}}\right) .
\end{aligned}
$$

By linearity, it suffices to prove convergence of the DDFV optimized Schwarz algorithm (3.5) to 0 . We have constructed $\left(u_{\mathcal{T}_{j}}^{\infty}, \psi_{\mathcal{T}_{j}}^{\infty}\right)$ from the solution $u_{\mathcal{T}}$ of the DDFV scheme (3.4) on $\Omega$ such that

$$
\mathcal{L}_{\Omega_{j}, \Gamma}^{\mathcal{T}_{j}}\left(u_{\mathcal{T}_{j}}^{\infty}, \psi_{\mathcal{T}_{j}}^{\infty} f_{\mathcal{T}_{j}}, h_{\mathcal{T}_{j}}^{\infty}\right)=0
$$

Observe that the errors $e_{\mathcal{T}_{j}}^{\ell+1}=u_{\mathcal{T}_{j}}^{\ell+1}-u_{\mathcal{T}_{j}}^{\infty} \Psi_{\mathcal{T}_{j}}^{\ell+1}=\psi_{\mathcal{T}_{j}}^{\ell+1}-\psi_{\mathcal{T}_{j}}^{\infty}$ satisfy

$$
\mathcal{L}_{\Omega_{j}, \Gamma}^{\mathcal{T}_{j}}\left(e_{\mathcal{T}_{j}}^{\ell+1}, \Psi_{\mathcal{T}_{j}}^{\ell+1}, 0, H_{\mathcal{T}_{j}}^{\ell}\right)=0
$$

with

$$
\begin{aligned}
& H_{\mathrm{K}^{*}}^{\ell}=-\Psi_{\mathrm{L}^{*}}^{\ell}+\Lambda_{\mathrm{L}^{*}}^{\partial \mathfrak{M}_{i, \Gamma}^{*}}\left(e_{\partial \mathfrak{M}_{i, \Gamma}^{\ell}}^{\ell}\right), \forall \mathrm{K}^{*} \in \partial \mathfrak{M}_{j, \Gamma}^{*} \text { and } \mathrm{L}^{*} \in \partial \mathfrak{M}_{i, \Gamma}^{*} \text { s. t. } x_{\mathrm{K}^{*}}=x_{\mathrm{L}^{*}}, \\
& H_{\mathrm{L}}^{\ell}=-\frac{1}{m_{\sigma}}\left(A_{\mathrm{D}} \nabla^{\mathrm{D}} e_{\mathcal{T}_{i}}^{\ell}, \mathbf{N}_{\mathrm{KL}}\right)+\Lambda_{\mathrm{L}}^{\partial \mathfrak{M}_{i, \Gamma}}\left(e_{\partial \mathfrak{M}_{i, \Gamma}}^{\ell}\right), \quad \forall \mathrm{L} \in \partial \mathfrak{M}_{i, \Gamma} .
\end{aligned}
$$

An a priori estimate using discrete duality leads to

$$
\begin{aligned}
2 \sum_{\mathrm{D} \in \mathfrak{D}_{j}} m_{\mathrm{D}}\left(A_{\mathrm{D}} \nabla^{\mathrm{D}} e_{\mathcal{T}_{j}}^{\ell+1}, \nabla^{\mathrm{D}} e_{\mathcal{T}_{j}^{\ell}}^{\ell+1}\right) & \\
& -\sum_{\mathrm{L} \in \partial \mathfrak{M}_{j, \Gamma}}\left(A_{\mathrm{D}} \nabla^{\mathrm{D}} e_{\mathcal{T}_{j}^{\ell+1}}^{\ell+1}, \mathbf{N}_{\mathrm{KL}}\right) e_{\mathrm{L}}^{\ell+1}-\sum_{\mathrm{K}^{*} \in \partial \mathfrak{M}_{j, \Gamma}^{*}} m_{\sigma_{\mathrm{K}^{*}}} \Psi_{\mathrm{K}^{*}}^{\ell+1} e_{\mathrm{K}^{*}}^{\ell+1} \\
& +\sum_{\mathrm{K} \in \mathfrak{M}_{j}} m_{\mathrm{K}} \eta_{\mathrm{K}}\left(e_{\mathrm{K}}^{\ell+1}\right)^{2}+\sum_{\mathrm{K}^{*} \in \mathfrak{M}_{j}^{*} \cup \partial \mathfrak{M}_{j, \Gamma}^{*}} m_{\mathrm{K}^{*}} \eta_{\mathrm{K}^{*}}\left(e_{\mathrm{K}^{*}}^{\ell+1}\right)^{2}=0 .
\end{aligned}
$$


Using the scalar product defined by $\left(\Lambda^{\partial \mathfrak{M}_{j, \Gamma}}\right)^{-1}$, we get

$$
\begin{aligned}
-\sum_{\mathrm{L} \in \partial \mathfrak{M}_{j, \Gamma}}\left(A_{\mathrm{D}} \nabla^{\mathrm{D}} e_{\mathcal{T}_{j}}^{\ell+1}, \mathbf{N}_{\mathrm{KL}}\right) e_{\mathrm{L}}^{\ell+1} & =\left(\left(A_{\mathfrak{D}} \nabla^{\mathfrak{D}} e_{\mathcal{T}_{j}}^{\ell+1}, \mathbf{n}_{j}\right), e_{\partial \mathfrak{M}_{j, \Gamma}^{\ell+1}}\right) \\
& =\left(\left(A_{\mathcal{D}} \nabla^{\mathfrak{D}} e_{\mathcal{T}_{j}^{\ell+1}}^{\ell+\mathbf{n}_{j}}\right),\left(\Lambda^{\partial \mathfrak{M}_{j, \Gamma}}\right)^{-1}\left(\Lambda^{\partial \mathfrak{M}_{j, \Gamma}}\left(e_{\partial \mathfrak{M}_{j, \Gamma}^{\ell+1}}^{\ell+1}\right)\right)\right) \\
& =\left(\left(A_{\mathcal{D}} \nabla^{\mathcal{D}} e_{\mathcal{T}_{j}^{\ell+1}}^{\ell+\mathbf{n}_{j}}\right), \Lambda^{\partial \mathfrak{M}_{j, \Gamma}}\left(e_{\partial \mathfrak{M}_{j, \Gamma}^{\ell+1}}^{\ell+1}\right)_{\left(\Lambda^{\partial \mathfrak{M}_{j, \Gamma}}\right)^{-1}} .\right.
\end{aligned}
$$

Using the same trick as at the continuous level, the formula $-a b=\frac{1}{4}\left((a-b)^{2}-(a+b)^{2}\right)$ implies

$$
\begin{aligned}
& -\sum_{\mathrm{L} \in \partial \mathfrak{M}_{j, \Gamma}}\left(A_{\mathrm{D}} \nabla^{\mathrm{D}} e_{\mathcal{T}_{j}}^{\ell+1}, \mathbf{N}_{\mathrm{KL}}\right) e_{\mathrm{L}}^{\ell+1}=\frac{1}{4}\left\|-\left(A_{\mathfrak{D}} \nabla^{\mathfrak{D}} e_{\mathcal{T}_{j}^{\ell}}^{\ell+1}, \mathbf{n}_{j}\right)+\Lambda^{\partial \mathfrak{M}_{j, \Gamma}\left(e_{\partial \mathfrak{M}_{j, \Gamma}^{\ell+1}}^{\ell+1}\right)}\right\|_{(\Lambda}^{2} \mathfrak{M}_{j, \Gamma}-1 \\
& -\frac{1}{4} \|\left(A_{\mathfrak{D}} \nabla^{\mathfrak{D}} e_{\mathcal{T}_{j}}^{\ell+1}, \mathbf{n}_{j}\right)+\Lambda^{\partial \mathfrak{M}_{j, \Gamma}}\left(e_{\partial \mathfrak{M}_{j, \Gamma}^{\ell+1}}^{\ell+1} \|_{\left(\Lambda^{\partial \mathfrak{M}_{j, \Gamma}-1}\right.}^{2} .\right.
\end{aligned}
$$

We can now use the Ventcell transmission conditions to replace the last term,

$$
\begin{aligned}
&-\sum_{\mathbf{L} \in \partial \mathfrak{M}_{j, \Gamma}}\left(A_{\mathrm{D}} \nabla^{\mathrm{D}} e_{\mathcal{T}_{j}}^{\ell+1}, \mathbf{N}_{\mathrm{KL}}\right) e_{\mathrm{L}}^{\ell+1}=\frac{1}{4}\left\|-\left(A_{\mathfrak{D}} \nabla^{\mathfrak{D}} e_{\mathcal{T}_{j}}^{\ell+1}, \mathbf{n}_{j}\right)+\Lambda^{\partial \mathfrak{M}_{j, \Gamma}}\left(e_{\partial \mathfrak{M}_{j, \Gamma}^{\ell+1}}^{\ell+1}\right)\right\|_{(\Lambda}^{\left.\partial \mathfrak{M}_{j, \Gamma}\right)^{-1}} \\
&-\frac{1}{4}\left\|-\left(A_{\mathfrak{D}} \nabla^{\mathfrak{D}} e_{\mathcal{T}_{i}}^{\ell}, \mathbf{n}_{j}\right)+\Lambda^{\partial \mathfrak{M}_{j, \Gamma}}\left(e_{\partial \mathfrak{M}_{i, \Gamma}}^{\ell}\right)\right\|_{(\Lambda}^{\partial \mathfrak{M}_{j, \Gamma}-1} \\
& 2
\end{aligned}
$$

Similarly, we also obtain on the dual mesh

$$
\begin{aligned}
& -\sum_{\mathrm{K}^{*} \in \partial \mathfrak{M}_{j, \Gamma}^{*}} m_{\sigma_{\mathrm{K}^{*}}} \Psi_{\mathrm{K}^{*}}^{\ell+1} e_{\mathrm{K}^{*}}^{\ell+1}=\frac{1}{4} \|-\Psi_{\mathcal{T}_{j}}^{\ell+1}+\Lambda^{\partial \mathfrak{M}_{j, \Gamma}^{*}}\left(e_{\partial \mathfrak{M}_{j, \Gamma}^{\ell+1}}^{\ell+1} \|_{(\Lambda}^{2} \mathfrak{M}_{j, \Gamma}^{*}\right)^{-1} \\
& -\frac{1}{4}\left\|-\Psi_{\mathcal{T}_{i}}^{\ell}+\Lambda^{\partial \mathfrak{M}_{j, \Gamma}^{*}}\left(e_{\partial \mathfrak{M}_{i, \Gamma}^{\ell}}^{\ell}\right)\right\|_{\left(\Lambda^{\left.\partial \mathfrak{M}_{j, \Gamma}^{*}\right)^{-1}}\right.}^{2} .
\end{aligned}
$$

Summing over $\ell=0, \cdots, \ell_{\max }-1$ and $j=1,2$, we get

$$
\begin{aligned}
& 2 \sum_{\ell=0}^{\ell_{\max }-1} \sum_{j=1,2} \sum_{\mathfrak{D} \in \mathfrak{D}_{j}} m_{\mathrm{D}}\left(A_{\mathrm{D}} \nabla^{\mathrm{D}} e_{\mathcal{T}_{j}}^{\ell+1}, \nabla^{\mathrm{D}} e_{\mathcal{T}_{j}}^{\ell+1}\right) \\
& +\sum_{\ell=0}^{\ell_{\max }-1} \sum_{j=1,2} \sum_{\mathrm{K} \in \mathfrak{M}_{j}} m_{\mathrm{K}} \eta_{\mathrm{K}}\left(e_{\mathrm{K}}^{\ell+1}\right)^{2}+\sum_{\ell=0}^{\ell_{\max }-1} \sum_{j=1,2} \sum_{\mathrm{K}^{*} \in \mathfrak{M}_{j}^{*} \cup \partial \mathfrak{M}_{j, \Gamma}^{*}} m_{\mathrm{K}^{*}} \eta_{\mathrm{K}^{*}}\left(e_{\mathrm{K}^{*}}^{\ell+1}\right)^{2} \\
& +\frac{1}{4} \sum_{j=1,2}\left\|-\left(A_{\mathfrak{D}} \nabla^{\mathfrak{D}} e_{\mathcal{T}_{j}}^{\ell_{\max }}, \mathbf{n}_{j}\right)+\Lambda^{\partial \mathfrak{M}_{j, \Gamma}}\left(e_{\partial \mathfrak{M}_{j, \Gamma}^{\ell_{\max }}}\right)\right\|_{\left(\Lambda^{\partial \mathfrak{M}_{j, \Gamma}}\right)^{-1}}^{2} \\
& +\sum_{j=1,2} \frac{1}{4}\left\|-\Psi_{\mathcal{T}_{j}}^{\ell_{\max }}+\Lambda^{\partial \mathfrak{M}_{j, \Gamma}^{*}}\left(e_{\partial \mathfrak{M}_{j, \Gamma}^{*}}^{\ell_{\max }}\right)\right\|_{(\Lambda}^{2}{ }_{\left(\mathfrak{M}_{j, \Gamma}^{*}\right)^{-1}}^{2}
\end{aligned}
$$



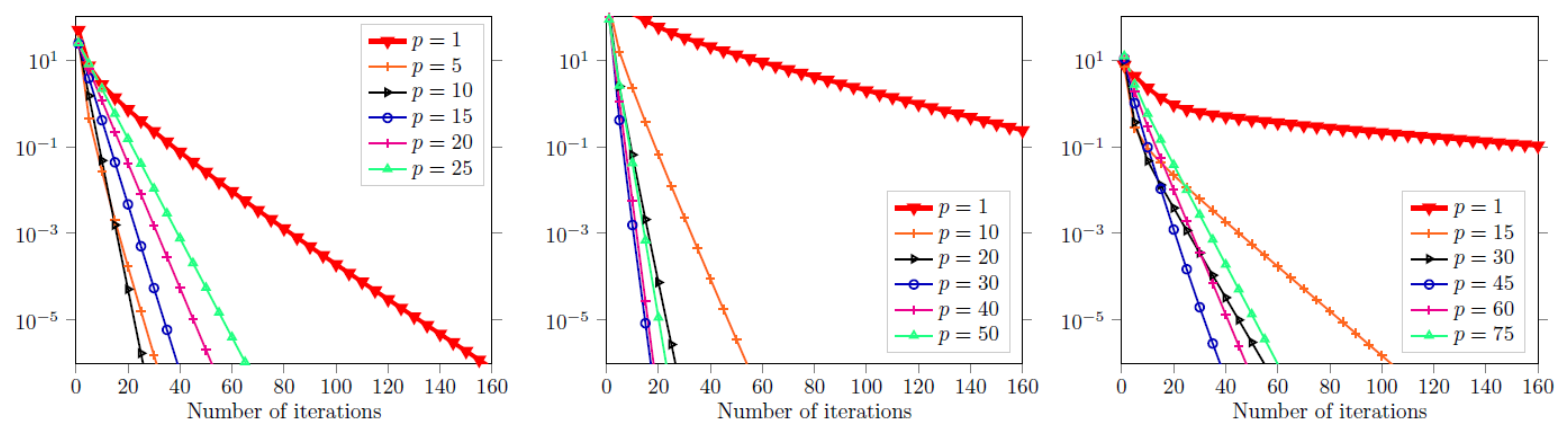

FIgURE 6. Convergence of the DDFV Schwarz algorithm for various parameter choices $p$ with $h=2^{-3}$ and random initial guess. Left: $A_{x x}=A_{y y}=1, A_{x y}=0$. Middle: $A_{x x}=16, A_{y y}=1, A_{x y}=0$. Right: $A_{x x}=1, A_{y y}=16, A_{x y}=0$.

$$
\begin{aligned}
& =\sum_{j=1,2} \frac{1}{4}\left\|-\left(A_{\mathfrak{D}} \nabla^{\mathfrak{D}} e_{\mathcal{T}_{j}}^{0}, \mathbf{n}_{j}\right)+\Lambda^{\partial \mathfrak{M}_{j, \Gamma}}\left(e_{\partial \mathfrak{M}_{j, \Gamma}}^{0}\right)\right\|_{\left(\Lambda^{\partial \mathfrak{M}_{j, \Gamma}}\right)^{-1}}^{2} \\
& +\sum_{j=1,2} \frac{1}{4} \|-\Psi_{\mathcal{T}_{j}}^{0}+\Lambda^{\left.\partial \mathfrak{M}_{j, \Gamma}^{*}\left(e_{\partial \mathfrak{M}_{j, \Gamma}^{*}}^{0}\right) \|_{(\Lambda}^{2} \mathfrak{M M}_{j, \Gamma}^{*}\right)^{-1}} \cdot
\end{aligned}
$$

This shows that also in the discrete case, the total energy stays bounded as the iteration index $\ell$ goes to infinity, and hence the algorithm converges.

\section{Numerical experiments}

We now investigate the influence of the anisotropy on the optimized Schwarz algorithm discretized by DDFV $(3.8,3.9)$ numerically. We start with numerical experiments for a rectangular domain decomposed into two rectangular subdomains, which corresponds precisely to our analysis, and allows us to illustrate when the bounded domain analysis is important, and why it is essential for performance to have the appropriate optimized choice of the Robin parameter in the anisotropic case. We then also investigate cases not covered by our analysis, namely domains which are not rectangular, and also decompositions into more than two subdomains.

4.1. Rectangular domain with two subdomains. We consider the domain $\Omega=(-1,1) \times(0,1)$ with the two subdomains $\Omega_{1}=(-1,0) \times(0,1)$ and $\Omega_{2}=(0,1) \times(0,1)$. We first compare the convergence on conforming Cartesian meshes using the mesh size $h=\frac{1}{8}$ for $\eta=1$ and $A_{x x}=A_{y y}=1, A_{x y}=0$ (the Laplacian), to the anisotropic cases with $A_{x x}=16, A_{y y}=1$, $A_{x y}=0$, and $A_{x x}=1, A_{y y}=16, A_{x y}=0$. We simulate directly the error equations, measure the error in the discrete $L^{2}$ norm over primal and dual unknowns, and start using a random initial guess, which is important to test the algorithm using all possible frequencies in the error, for a detailed explanation, see [19, Section 5.1, last paragraph]. We show in Figure 6 how the error decreases over the iterations for different choices of the optimization parameter $p$. We see that in all cases a good choice of $p$ leads to fast convergence, and the value of the best $p$ is influenced by the anisotropy. We also observe that the anisotropic case is harder to 


\begin{tabular}{|c|c|c|c|c|c|c|}
\hline & $p_{\infty}^{*}$ & iter & $p_{L}^{*}$ & iter & $p_{\text {num }}^{*}$ & iter \\
\hline Mesh & \multicolumn{6}{|c|}{$A=\mathrm{Id}$} \\
\hline $2^{-3}$ & 9.11 & 43 & 9.12 & 43 & 8.34 & 39 \\
\hline $2^{-4}$ & 12.87 & 60 & 12.89 & 60 & 11.81 & 55 \\
\hline $2^{-5}$ & 18.21 & 85 & 18.23 & 85 & 16.43 & 76 \\
\hline $2^{-6}$ & 25.75 & 120 & 25.78 & 120 & 22.42 & 105 \\
\hline & \multicolumn{6}{|c|}{$A_{x x}=16, A_{y y}=1$} \\
\hline $2^{-3}$ & 36.43 & 28 & 44.26 & 35 & 35.17 & 27 \\
\hline $2^{-4}$ & 51.50 & 40 & 62.57 & 49 & 49.62 & 39 \\
\hline $2^{-5}$ & 72.82 & 59 & 88.48 & 72 & 69.46 & 56 \\
\hline $2^{-6}$ & 102.99 & 82 & 125.13 & 100 & 96.33 & 77 \\
\hline & \multicolumn{6}{|c|}{$A_{x x}=1, A_{y y}=16$} \\
\hline $2^{-3}$ & 35.60 & 95 & 35.60 & 95 & 49.09 & 73 \\
\hline $2^{-4}$ & 50.34 & 136 & 50.34 & 136 & 74.44 & 95 \\
\hline $2^{-5}$ & 71.20 & 186 & 71.20 & 186 & 104.99 & 129 \\
\hline $2^{-6}$ & 100.69 & 254 & 100.69 & 254 & 140.51 & 185 \\
\hline
\end{tabular}

TABLE 1. Optimized Robin parameters $p_{\infty}^{*}$ and $p_{L}^{*}$ using $k_{\min }=\pi$ and $k_{\max }=\frac{\pi}{h}$, and $p_{\text {num }}^{*}$ performing best in numerical experiments, together with the corresponding number of iterations to reach an error reduction of $1 e-12$.

solve for the method than the Laplacian case for the generic parameter choice $p=1$, but good convergence is restored for a well chosen parameter. We show in Table 1 a detailed comparison of our asymptotically predicted optimized Robin parameters $p_{\infty}^{*}$ for the unbounded domain analysis, $p_{L}^{*}$ from the bounded domain analysis, and $p_{n u m}^{*}$ that worked numerically best, i.e. reaching the very small tolerance of $1 e-12$ in the smallest number of iterations. We also show the corresponding number of iterations to achieve this tolerance for each parameter choice. There are two interesting observations: first, in the case of the Laplacian, the unbounded domain analysis gives a Robin parameter $p_{\infty}^{*}$ which is very similar to the bounded domain Robin parameter $p_{L}^{*}$, and the same holds also for the anisotropic case $A_{x x}=1$ and $A_{y y}=16$. However, when $A_{x x}=16$ and $A_{y y}=1$, this is not the case any more, because due to the strong diffusion in the $x$ direction, the homogeneous Dirichlet boundary conditions at $x=-1$ and $x=1$ influence the solution substantially, and thus the boundedness needs to be taken into account to determine the optimized parameter $p_{L}^{*}$, which is different from $p_{\infty}^{*}$. Second, in the case of the Laplacian, the analysis also predicts well the parameter $p_{n u m}^{*}$ that works best, but in the anisotropic cases, even though the asymptotic behavior of the optimized parameter is well captured, in the case $A_{x x}=16$ and $A_{y y}=1$ the continuous prediction is a bit too large, and the bounded continuous analysis which should be more accurate is actually less accurate. In the case $A_{x x}=1$ and $A_{y y}=16$ the prediction of the continuous analysis is a bit too small. To quantify this, one would need a fully discrete analysis, which is beyond the scope of the present paper and will be the subject of further studies.

We show next in Table 2 the corresponding results for the optimized Ventcell parameters. We observe again as in the case of the Robin parameters that the asymptotically best parameters 


\begin{tabular}{|c||c|c|c||c|c|c||c||c|c|c|}
\hline & $p_{\infty}^{*}$ & $q_{\infty}^{*}$ & iter & $p_{L}^{*}$ & $q_{L}^{*}$ & iter & $p_{\text {num }}^{*}$ & $q_{\text {num }}^{*}$ & iter \\
\hline Mesh & \multicolumn{10}{|c|}{$A=$ Id } \\
\hline $2^{-3}$ & 3.6870 & 0.0439 & 19 & 3.6959 & 0.0439 & 19 & 4.0220 & 0.04699 & 18 \\
\hline $2^{-4}$ & 4.4898 & 0.0269 & 20 & 4.4998 & 0.0269 & 20 & 4.5815 & 0.02878 & 20 \\
\hline $2^{-5}$ & 5.4069 & 0.0163 & 24 & 5.4185 & 0.0163 & 24 & 5.3173 & 0.01745 & 24 \\
\hline $2^{-6}$ & 6.4718 & 0.0097 & 29 & 6.4853 & 0.0097 & 29 & 6.1922 & 0.01063 & 27 \\
\hline \multicolumn{10}{||}{} & \multicolumn{10}{|c|}{$A_{x x}=16, A_{y y}=1$} \\
\hline $2^{-3}$ & 14.7479 & 0.1757 & 16 & 20.7545 & 0.1693 & 14 & 19.1678 & 0.18871 & 11 \\
\hline $2^{-4}$ & 17.9591 & 0.1077 & 20 & 24.6158 & 0.1059 & 17 & 22.1708 & 0.1249 & 15 \\
\hline $2^{-5}$ & 21.6275 & 0.0651 & 24 & 29.2925 & 0.0645 & 22 & 26.2975 & 0.07849 & 19 \\
\hline $2^{-6}$ & 25.8870 & 0.0390 & 30 & 34.8627 & 0.0388 & 27 & 30.8763 & 0.04811 & 24 \\
\hline \multicolumn{1}{|||||}{} & \multicolumn{10}{|c|}{$A_{x x}=1, A_{y y}=16$} \\
\hline $2^{-3}$ & 14.1316 & 0.0111 & 59 & 14.1316 & 0.0111 & 59 & 35.9474 & 0.00699 & 55 \\
\hline $2^{-4}$ & 17.2871 & 0.0068 & 56 & 17.2871 & 0.0068 & 56 & 42.0509 & 0.00542 & 55 \\
\hline $2^{-5}$ & 20.8638 & 0.0041 & 56 & 20.8638 & 0.0041 & 56 & 44.7826 & 0.00330 & 55 \\
\hline $2^{-6}$ & 24.9996 & 0.0025 & 66 & 24.9996 & 0.0025 & 66 & 44.8830 & 0.00184 & 54 \\
\hline
\end{tabular}

TABLE 2. Optimized Ventcell parameters $p_{\infty}^{*}, q_{\infty}^{*}, p_{L}^{*}$ and $q_{L}^{*}$ for $k_{\min }=\pi$ and $k_{\max }=\frac{\pi}{h}$, and $p_{\text {num }}^{*}, q_{\text {num }}^{*}$ performing best in numerical experiments, together with the corresponding number of iterations to reach an error reduction of $1 e-$ 12.

are predicted well, and the bounded domain analysis is important if $A_{x x}$ is large. For strong anisotropies, as in the Robin case, there is a certain difference in the constants that could only be explained with a fully discrete analysis, which would then however be limited to a particular mesh.

4.2. Non-Rectangular domains with non-matching grids. We now show that the continuous analysis allows us to determine optimized parameters that work well also in more realistic situations, where we have non-matching grids and non-rectangular geometries and meshes, namely the two experiments shown in Figure 7. We start with a zero initial guess. For the problem on the left, we use a diagonal diffusion matrix with $A_{x x}=16$ and $A_{y y}=1$ and the source function $f(x, y)=e^{-(x+0.5)^{2}-(y-0.5)^{2}}$. We show in Table 3 how many iterations our code needs to converge to an accuracy of $1 \%$ with respect to a converged solution when using $p=1, q=0, p=300, q=0$, compared to using the optimized Robin parameter $p_{\infty}^{*}=63.07$ and Ventcell parameters $p_{\infty}^{*}=20.04$ and $q_{\infty}^{*}=0.0803$, which we obtained from our continuous analysis using the smaller mesh size in the estimate for $k_{\max }=\pi / h$. We clearly see that using the parameters predicted by the continuous analysis leads to great savings in the number of iterations needed, and this without changing the computational cost per iteration. Similar results we obtained also for the example on the right in Figure 7, where we now used the fully anisotropic diffusion matrix $A=\left[\begin{array}{cc}16 & 0.5 \\ 0.5 & 1\end{array}\right]$ and the same source function as before, see 

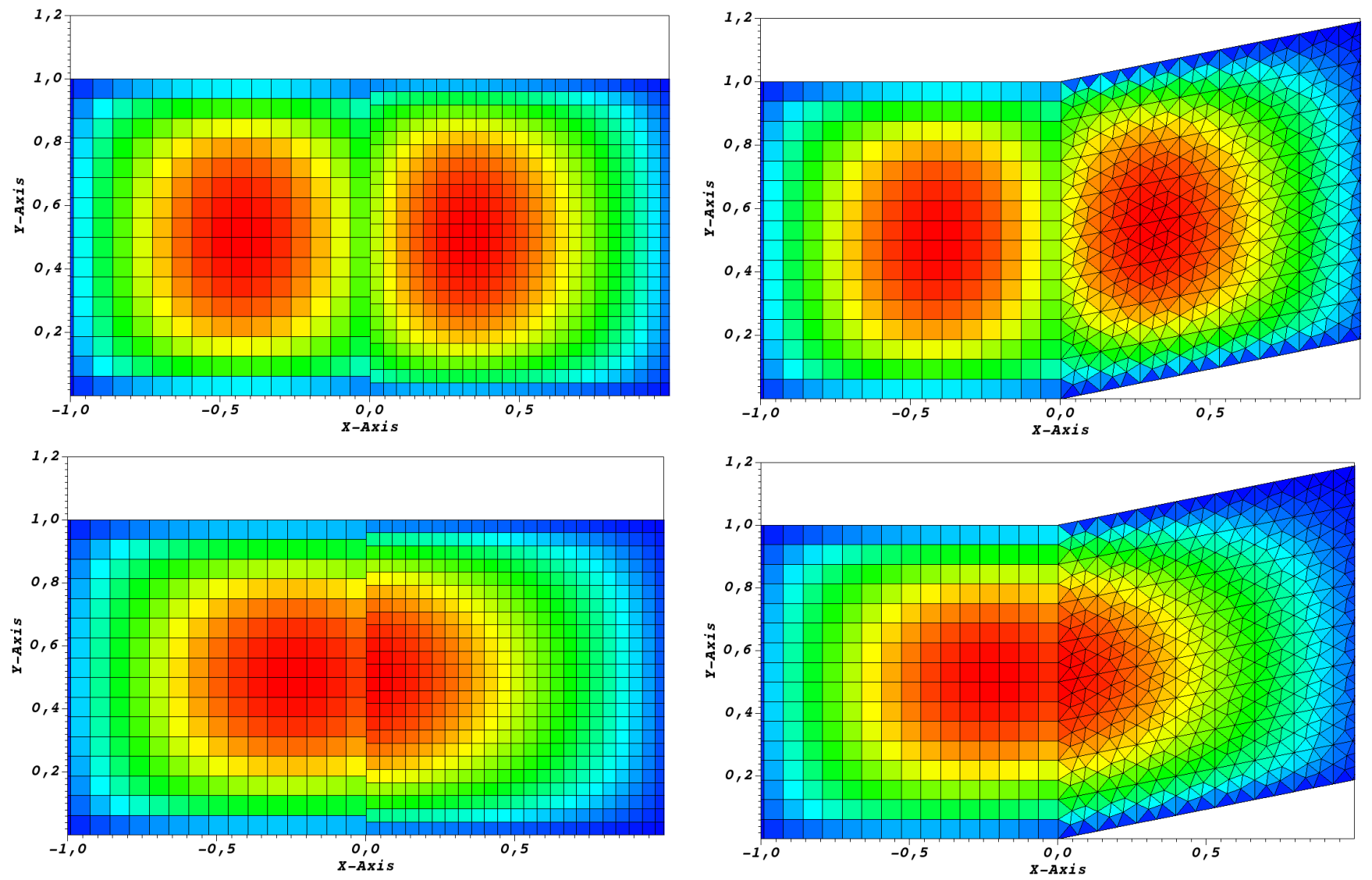

FIGURE 7. First and third iteration of the DDFV optimized Schwarz method with Ventcell transmission conditions. Left: Nonconforming mesh with diagonal $A$. Right. Irregular domain and mesh with a fully anisotropic $A$.

\begin{tabular}{|c|c|c|c|c|}
\hline Problem & $p=1, q=0$ & $p=300, q=0$ & $p=p_{\infty,}^{*}, q=0$ & $p=p_{\infty,}^{*}, q=q_{\infty}^{*}$ \\
\hline left & 33 & 35 & 8 & 3 \\
\hline right & 37 & 34 & 7 & 3 \\
\hline
\end{tabular}

TABLE 3. Number of iterations needed when solving the left and right problem in Figure 7 using the DDFV Schwarz algorithm.

Table 3. The optimized parameters predicted by our continuous analysis were $p_{\infty}^{*}=51.5$ for the Robin case, and $p_{\infty}^{*}=17.96$ and $q_{\infty}^{*}=0.1077$ for the Ventcell case. Again the predicted parameters lead to important savings.

4.3. Layered multidomain decompositions. We finally show an experiment where we decompose a layer of variable anisotropic diffusion into many subdomains. The domain, together with the source term $e^{-1.25\left(0.1(x-4.0)^{2}+(y-0.5)^{2}\right)}$, is shown in Figure 8, and we use $\eta=1$ in the following experiments. 


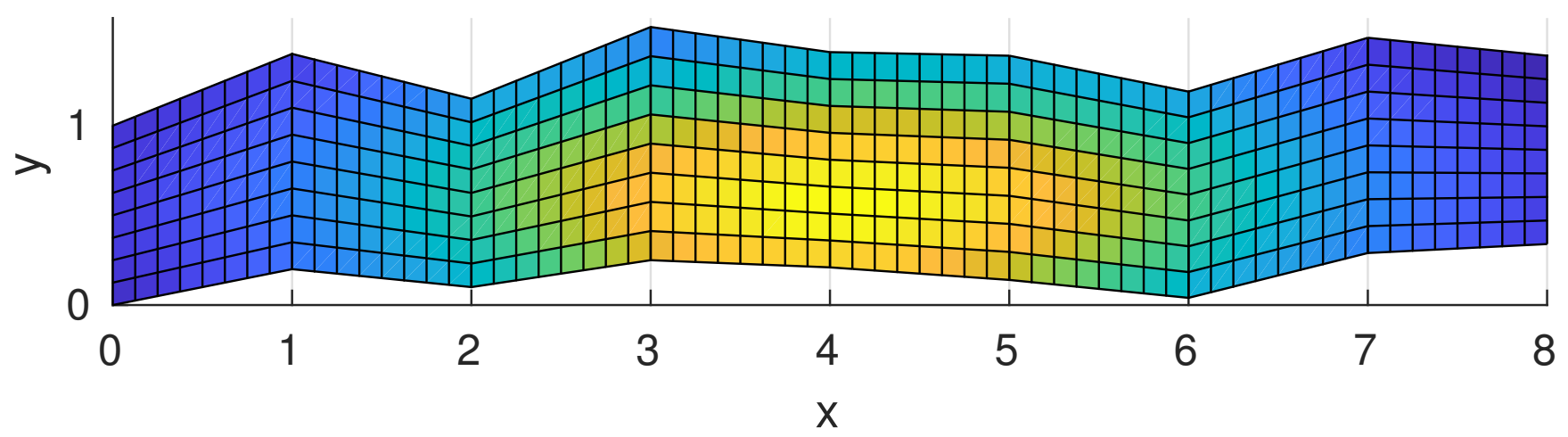

FigURE 8. Layer domain for the many subdomain decomposition and source term for our experiments.

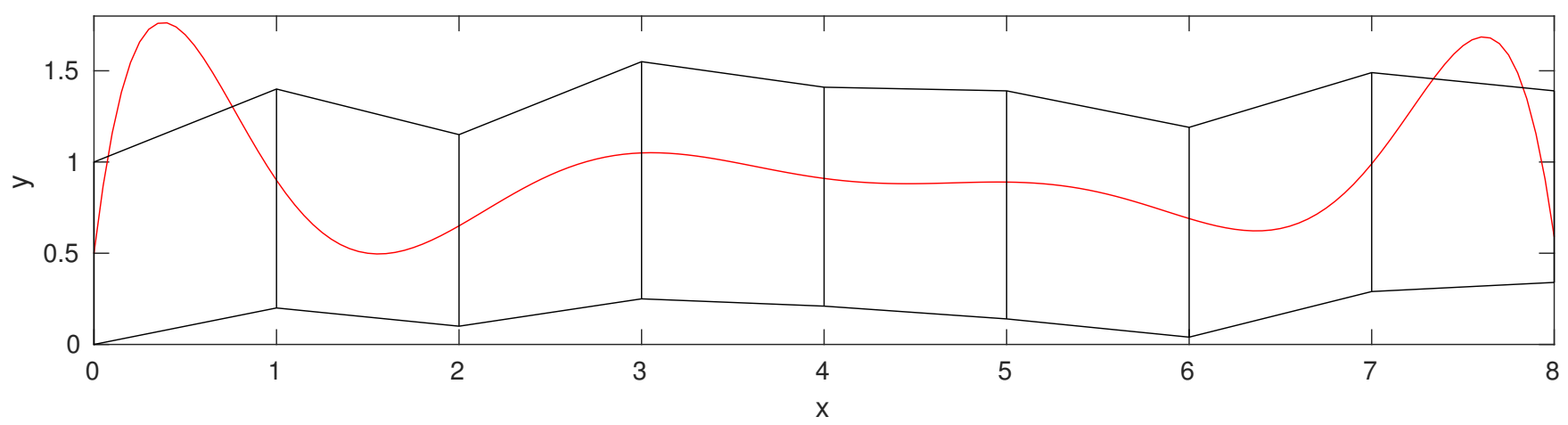

FiguRE 9. Decomposition of the layer domain into 8 subdomains, and the Lagrange interpolation polynomial $\phi(x)$.

The variable diffusion matrix $A$ is defined as follows: we first define the Lagrange interpolation polynomial $\phi(x)$ of degree 8 shown in red in Figure 9, which passes through the 9 points $\left(x_{i}, y_{i}\right), i=0,1, \ldots, 8$ on the 9 boundaries of the subdomains, $x_{i}=i, y_{0}=0.5, y_{1}=0.9$, $y_{2}=0.65, y_{3}=1.05, y_{4}=0.91, y_{5}=0.89, y_{6}=0.69, y_{7}=0.99, y_{8}=0.59$, also shown in Figure 9. We then define for each point of the curve given by the Lagrange interpolation polynomial the normalized gradient and tangent vector

$$
\boldsymbol{n}(x):=\frac{1}{\sqrt{1+\left(\phi^{\prime}(x)\right)^{2}}}\left(1, \phi^{\prime}(x)\right)^{T}, \quad \boldsymbol{t}(x):=\frac{1}{\sqrt{1+\left(\phi^{\prime}(x)\right)^{2}}}\left(-\phi^{\prime}(x), 1\right)^{T} .
$$

We also define two coefficients $a_{1}$ and $a_{2}$ dependent on $x$ by

$$
\begin{aligned}
& a_{1}(x):=1.0(0.5 \tanh (12-4 x)+0.45 \tanh (4 x-20)+1), \\
& a_{2}(x):=0.9(0.5 \tanh (20-4 x)+0.45 \tanh (4 x-12)+0.1),
\end{aligned}
$$

which are shown in Figure 10 and will be used to determine the diffusion strength in the normal and tangential direction of the Lagrange interpolation polynomial. We now build the diffusion matrix $A$ such that the diffusion equals $a_{1}(x)$ in the direction $n(x)$ and $a_{2}(x)$ in the direction $t(x)$ for any point $x$, and $A$ is constant in $y$, see Figure 11. The anisotropic diffusion 


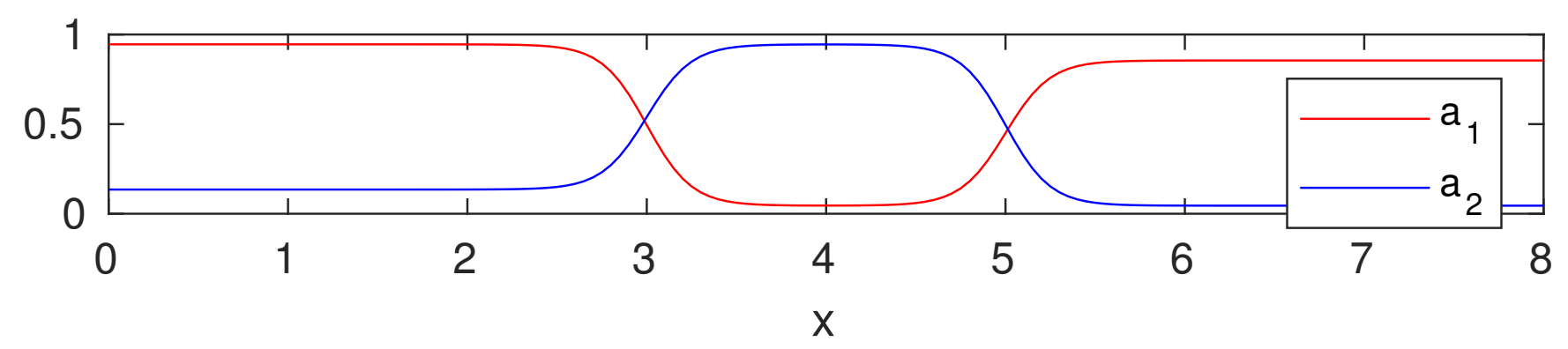

Figure 10. Coefficients $a_{1}$ and $a_{2}$ to determine the diffusion strength.

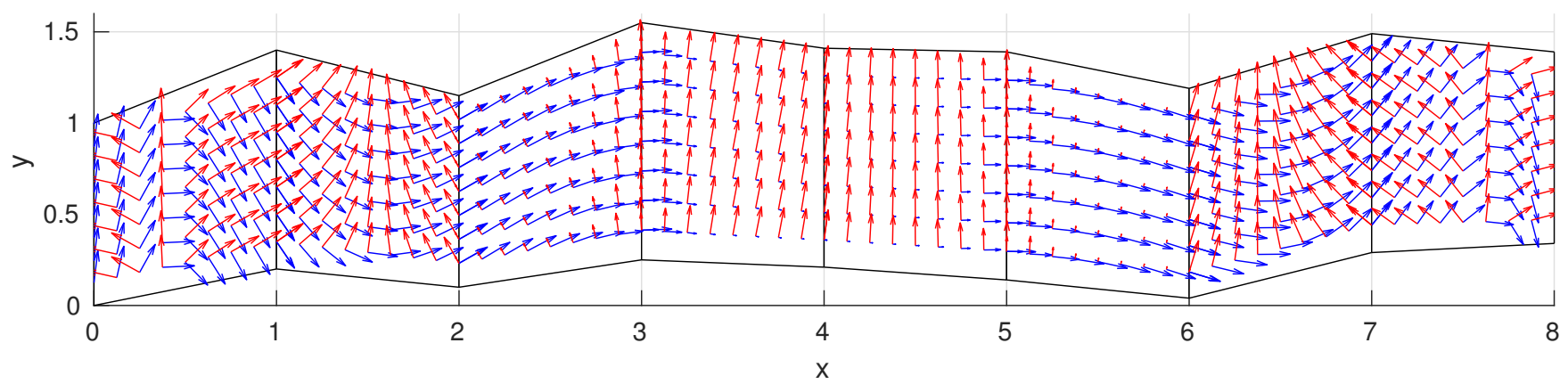

FIGURE 11. Vector fields $a_{1} n$ and $a_{2} t$ to build the anisotropic diffusion matrix.

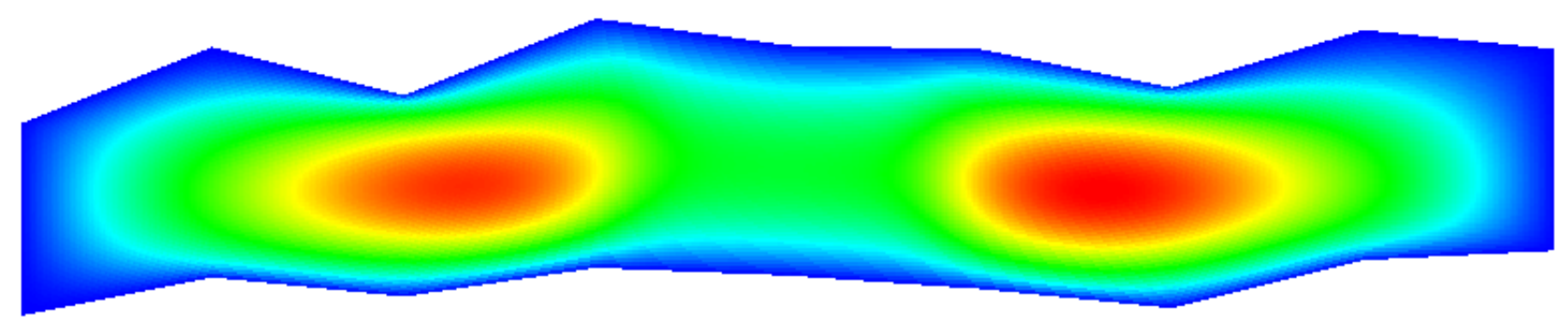

FIGURE 12. DDFV solution on the full domain: maximum solution value is 0.2958

matrix is thus given by the formula

$$
A(x):=a_{1}(x) \boldsymbol{n}(x) \boldsymbol{n}(x)^{T}+a_{2}(x) \boldsymbol{t}(x) \boldsymbol{t}(x)^{T} .
$$

We show the solution of this problem in Figure 12, where one can clearly see that the anisotropic diffusion generates two maxima, even though the source had only one. The solution was obtained using our new algorithm and 8 subdomains as indicated in Figure 9, with the optimized parameters from our two subdomain analysis shown in Table 4, starting with a zero initial guess. We show in Figure 13 the iterates $\ell=1,4,6,8,12,20$ to illustrate how the algorithm converges. We can see how the method first generates maxima in each subdomain, but very quickly identifies the true location of the maxima of the solution, and converges rapidly, without Krylov acceleration. We next also run our algorithm using only four subdomains, see Figure 14, and finally only two subdomains, see Figure 15. As expected, convergence is faster using less subdomains since we are just using a one level method, but we observe also 


\begin{tabular}{|c|c|c|}
\hline interface & $p$ & $q$ \\
\hline 1 & 2.099499 & $5.38921 \mathrm{E}-002$ \\
\hline 2 & 2.301869 & $4.77629 \mathrm{E}-002$ \\
\hline 3 & 2.454977 & $2.06458 \mathrm{E}-002$ \\
\hline 4 & 1.451507 & $6.15922 \mathrm{E}-003$ \\
\hline 5 & 2.535083 & $1.98993 \mathrm{E}-002$ \\
\hline 6 & 2.160908 & $5.18254 \mathrm{E}-002$ \\
\hline 7 & 2.099499 & $5.38921 \mathrm{E}-002$ \\
\hline
\end{tabular}

TABLE 4. Optimized Ventcell parameters for the layered multidomain decomposition used locally on the interfaces.

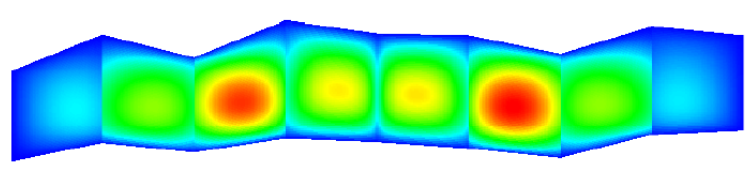

(a) $\ell=1, \max =0.1695$

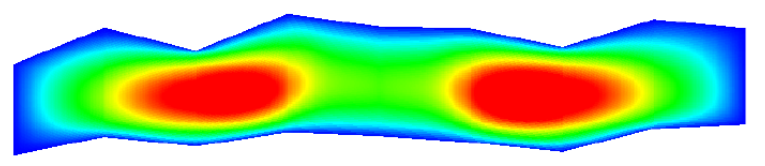

(c) $\ell=3, \max =0.279$

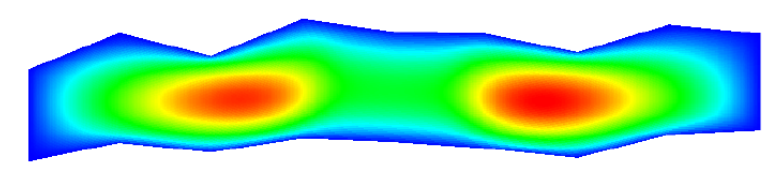

(e) $\ell=5, \max =0.2935$

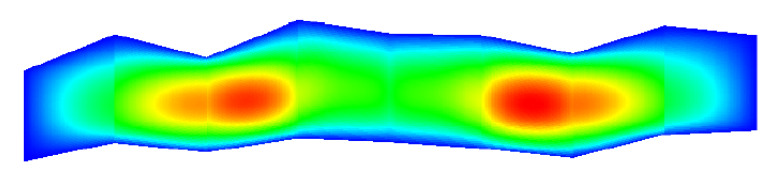

(b) $\ell=2, \max =0.2443$

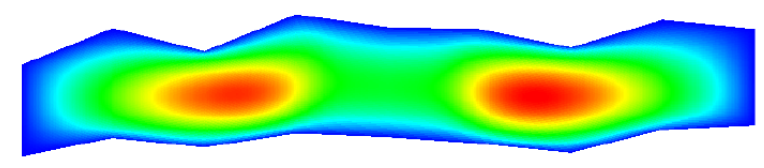

(d) $\ell=4, \max =0.2889$

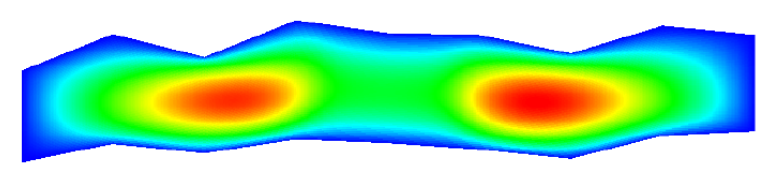

(f) $\ell=10, \max =0.2958$

FiguRE 13. Iterations $\ell=1,2,3,4,5,10$ for the 8 subdomain case, and maxima attained by the iterates.

that when cutting through the fast diffusion region in the middle only, the two maxima in the underlying solution are identified very rapidly.

\section{Conclusions}

We introduced a new, optimized DDFV Schwarz algorithm with general Ventcell transmission conditions for fully anisotropic diffusion, and showed that it is well posed and convergent using energy estimates and two subdomain decomposition. We also determined optimized Robin and Ventcell transmission conditions at the continuous level, both using the by now classical unbounded domain analysis, and a new technique which takes into account the boundedness of the domain. Our optimized transmission conditions lead to low iteration 


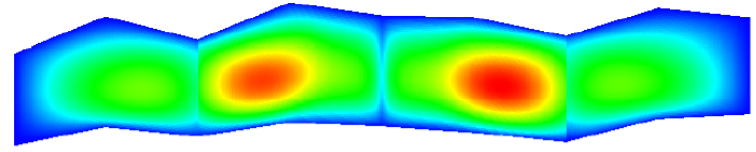

(a) $\ell=1, \max =0.2242$

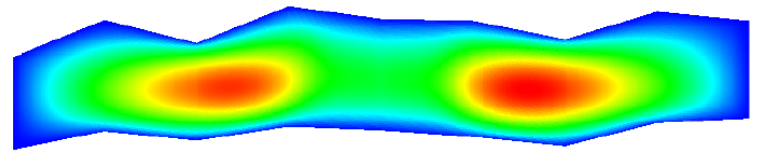

(c) $\ell=3, \max =0.2907$

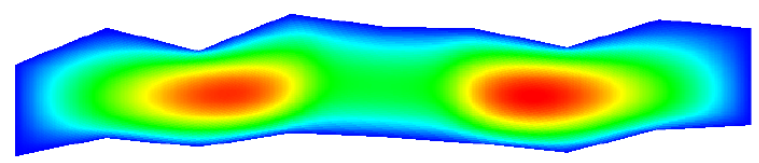

(e) $\ell=5, \max =0.2955$

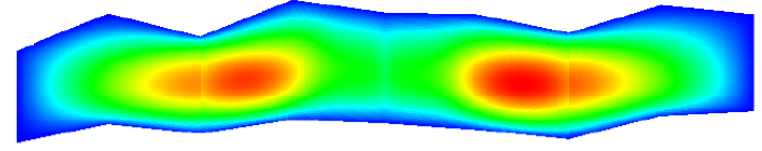

(b) $\ell=2, \max =0.2699$



(d) $\ell=4, \max =0.2942$

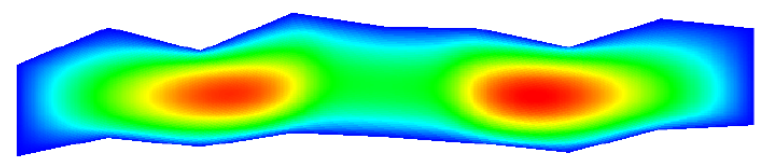

(f) $\ell=7, \max =0.2958$

FIgURE 14. Iterations $\ell=1,2,3,4,5,7$ for the 4 subdomain case, and maxima attained by the iterates.

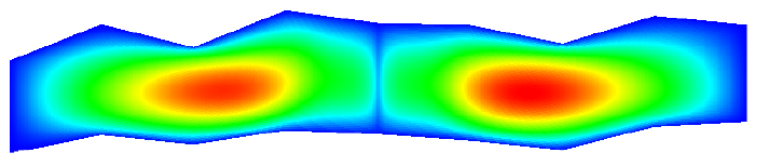

(a) $\ell=1, \max =0.2958$

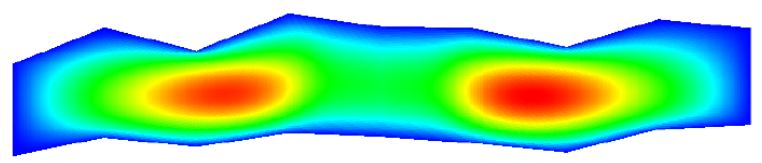

(c) $\ell=3, \max =0.2958$

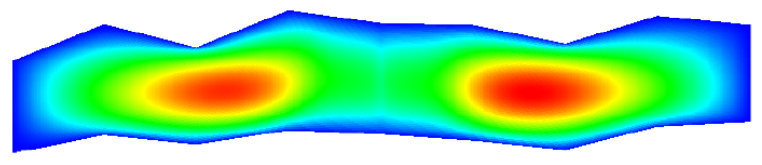

(b) $\ell=2, \max =0.2958$

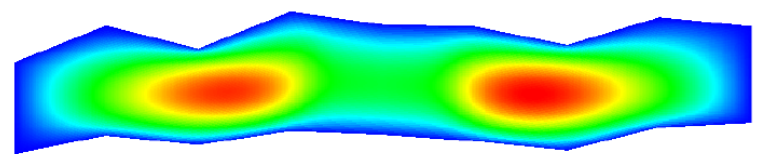

(d) $\ell=4, \max =0.2958$

FIGURE 15. Iterations $\ell=1,2,3,4$ for the 2 subdomain case, and maxima attained by the iterates.

counts for the algorithm, and for certain types of anisotropic diffusion, we showed that the bounded domain analysis is important. We also observed an interesting discrepancy in the case of large anisotropies between our continuous analysis and the discrete performance of the algorithm: while the asymptotically best parameter choice is well captured, there is a difference in the constants. We conjecture that this difference is related to the isotropic mesh size we use for the anisotropic diffusion model problems we solved, and to gain more insight into this phenomenon, we will have to embark on a fully discrete analysis.

While our analysis provides for the first time optimized transmission conditions for Schwarz methods for fully anisotropic diffusion problems with an appropriate discretization for such problems, this is only a first step in the development of scalable solvers for such problems. 
Two further main ingredients are needed for a scalable solver: the definition and analysis of the transmission conditions at cross points, and an adapted coarse space for anisotropic diffusion. For the simpler case of isotropic diffusion, an algorithm with Ventcell transmission conditions and cross points has been studied at the continuous level using energy estimates for rectangular decompositions in [34]. At the discrete level, a condition number estimate for a finite element discretizations of isotropic diffusion problems and Robin transmission conditions can be found in [33]. Two different consistent discretizations at cross points for finite element discretizations were derived and analyzed in [22], and optimized Robin parameters at cross points at the algebraic level were derived in [20], but classical energy estimates can not directly be used in the presence of cross points [21]. There is also to the best of the authors knowledge no study so far on efficient coarse spaces for anisotropic diffusion problems. In the case of the Poisson equation, very recently the combination of optimized transmission conditions with an adapted coarse space for optimized Schwarz methods led in an implementation in PETSc to substantially faster Schwarz methods than the default two level Schwarz solver in PETSc [23], and wall clock times are comparable to the multigrid solver implemented in PETSc. We are currently working on developing similar techniques also for the anisotropic diffusion case.

\section{References}

[1] B. Andreianov, F. Boyer, and F. Hubert. Discrete duality finite volume schemes for Leray-Lions type elliptic problems on general 2D-meshes. Num. Meth. for PDEs, 23(1):145-195, 2007.

[2] B. Andreianov, F. Hubert, and S. Krell. Benchmark 3D: a version of the DDFV scheme with cell/vertex unknowns on general meshes. In Finite Volumes for Complex Applications VI Problems \& Perspectives, pages 937-948. Springer, 2011.

[3] D. N. Arnold, R. S. Falk, and R. Winther. Finite element exterior calculus, homological techniques, and applications. Acta numerica, 15:1-155, 2006.

[4] D. Bennequin, M. J. Gander, and L. Halpern. A homographic best approximation problem with application to optimized Schwarz waveform relaxation. Mathematics of Computation, 78(265):185-223, 2009.

[5] F. Boyer and F. Hubert. Finite volume method for 2D linear and nonlinear elliptic problems with discontinuities. SIAM J. Numer. Anal., 46, 2008.

[6] F. Boyer, F. Hubert, and S. Krell. Non-overlapping Schwarz algorithm for solving 2D m-DDFV schemes. IMA Jour. Num. Anal., 30, 2009.

[7] F. Brezzi, K. Lipnikov, and V. Simoncini. A family of mimetic finite difference methods on polygonal and polyhedral meshes. Mathematical Models and Methods in Applied Sciences, 15(10):1533-1551, 2005.

[8] M. Chau, C. Tauber, and P. Spitéri. Parallel Schwarz alternating methods for anisotropic diffusion of speckled medical images. Numerical Algorithms, 51(1):85-114, 2009.

[9] Y. Coudiere, F. Hubert, and G. Manzini. A CeVeFE DDFV scheme for discontinuous anisotropic permeability tensors. In Finite Volumes for Complex Applications VI Problems \& Perspectives, pages 283-291. Springer, 2011.

[10] Y. Coudière, J.-P. Vila, and P. Villedieu. Convergence rate of a finite volume scheme for a two dimensional convection-diffusion problem. ESAIM: Mathematical Modelling and Numerical Analysis, 33(3):493-516, 1999.

[11] Z. Dai, Q. Du, and B. Liu. Schwarz alternating methods for anisotropic problems with prolate spheroid boundaries. SpringerPlus, 5(1):1423, 2016.

[12] K. Domelevo and P. Omnes. A finite volume method for the Laplace equation on almost arbitrary twodimensional grids. M2AN Math. Model. Numer. Anal., 39(6):1203-1249, 2005.

[13] J. Droniou, R. Eymard, and R. Herbin. Gradient schemes: Generic tools for the numerical analysis of diffusion equations. ESAIM: M2AN, 50(3):749-781, 2016.

[14] O. Dubois and M. Gander. Optimized Schwarz methods for a diffusion problem with discontinuous coefficient. Numer. Alg., 1:109-144, 2015. 
[15] R. Eymard, G. Henry, R. Herbin, F. Hubert, R. Klöfkorn, and G. Manzini. 3D benchmark on discretization schemes for anisotropic diffusion problems on general grids. In Finite Volumes for Complex Applications VI Problems \& Perspectives, pages 895-930. Springer, 2011.

[16] R. Eymard and J.-M. Hérard. Finite Volumes for Complex Applications V. John Wiley \& Sons, 2008.

[17] D. Furihata and T. Matsuo. Discrete variational derivative method: a structure-preserving numerical method for partial differential equations. Chapman and Hall/CRC Press, 2010.

[18] M. J. Gander. Optimized Schwarz Method. SIAM Journal on Numerical Analysis, 44(2):699-731, 2006.

[19] M. J. Gander. Schwarz methods over the course of time. Electron. Trans. Numer. Anal, 31(5):228-255, 2008.

[20] M. J. Gander and F. Kwok. Best Robin parameters for optimized Schwarz methods at cross points. SIAM Journal on Scientific Computing, 34(4):A1849-A1879, 2012.

[21] M. J. Gander and F. Kwok. On the applicability of Lions' energy estimates in the analysis of discrete optimized Schwarz methods with cross points. In Domain decomposition methods in science and engineering XX, pages 475-483. Springer, 2013.

[22] M. J. Gander and K. Santugini. Cross-points in domain decomposition methods with a finite element discretization. Electronic Transactions on Numerical Analysis, 45:219-240, 2016.

[23] M. J. Gander and S. Van Criekingen. New coarse corrections for optimized restricted additive Schwarz using PETSc. In Domain decomposition methods in science and engineering XXV. Springer, 2020.

[24] L. Gerardo-Giorda and F. Nataf. Optimized Schwarz methods for unsymmetric layered problems with strongly discontinuous and anisotropic coefficients. Journal of Numerical Mathematics, 13(4):265-294, 2005.

[25] E. Hairer, C. Lubich, and G. Wanner. Geometric numerical integration: structure-preserving algorithms for ordinary differential equations, volume 31. Springer Science \& Business Media, 2006.

[26] R. Herbin and F. Hubert. Benchmark on discretization schemes for anisotropic diffusion problems on general grids. In R. Eymard and J.-M. Hérard, editors, Finite Volumes for Complex Applications V, pages 659-692. John Wiley \& Sons, 2008.

[27] F. Hermeline. Approximation of diffusion operators with discontinuous tensor coefficients on distorted meshes. Comput. Methods Appl. Mech. Engrg., 192(16-18):1939-1959, 2003.

[28] J. Hyman, J. Morel, M. Shashkov, and S. Steinberg. Mimetic finite difference methods for diffusion equations. Computational Geosciences, 6(3):333-352, 2002.

[29] C. Japhet, Y. Maday, and F. Nataf. A new interface cement equilibrated mortar (NICEM) method with Robin interface conditions: the P1 finite element case. Mathematical Models and Methods in Applied Sciences, 23(12):2253-2292, 2013.

[30] C. Japhet, Y. Maday, and F. Nataf. A new interface cement equilibrated mortar method with Ventcel conditions. In Domain Decomposition Methods in Science and Engineering XXI, pages 377-385. Springer, 2014.

[31] T. Kashiwabara, C. M. Colciago, L. Dedè, and A. Quarteroni. Well-posedness, regularity, and convergence analysis of the finite element approximation of a generalized Robin boundary value problem. SIAM Journal on Numerical Analysis, 53(1):105-126, 2015.

[32] Y. A. Kuznetsov, O. Boiarkine, I. Kapyrin, and N. Yavich. Numerical analysis of a two-level preconditioner for the diffusion equation with an anisotropic diffusion tensor. Russian Journal of Numerical Analysis and Mathematical Modelling, 22(4):377-391, 2007.

[33] S. Loisel. Condition number estimates for the nonoverlapping optimized Schwarz method and the 2Lagrange multiplier method for general domains and cross points. SIAM Journal on Numerical Analysis, 51(6):3062-3083, 2013.

[34] F. Nataf. A Schwarz additive method with high order interface conditions and nonoverlapping subdomains. ESAIM: Mathematical Modelling and Numerical Analysis, 32(1):107-116, 1998.

[35] T. H. Ong and T.-T.-P. Hoang. Optimized Schwarz and finite element cell-centered method for heterogeneous anisotropic diffusion problems. Applied Numerical Mathematics, 151:380-401, 2020.

[36] L. F. Pavarino and S. Scacchi. Multilevel additive Schwarz preconditioners for the Bidomain reactiondiffusion system. SIAM Journal on Scientific Computing, 31(1):420-443, 2008.

[37] J. Szeftel. Calcul pseudo-différentiel et para-différentiel pour l'étude des conditions aux limites absorbantes et des propriétés qualitatives des EDP non linéaires. PhD thesis, Université Paris 13, France, 2004. 